ARTICLE

https://doi.org/10.1038/s41467-019-09734-5

\title{
AURKB as a target in non-small cell lung cancer with acquired resistance to anti-EGFR therapy
}

Jordi Bertran-Alamillo (1) 1, Valérie Cattan², Marie Schoumacher (1) 2, Jordi Codony-Servat', Ana Giménez-Capitán ${ }^{1}$, Frédérique Cantero², Mike Burbridge², Sonia Rodríguez ${ }^{1}$, Cristina Teixidó ${ }^{1,8}$,

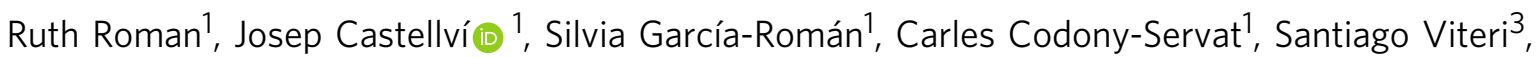
Andrés-Felipe Cardona ${ }^{4,5}$, Niki Karachaliou ${ }^{3}$, Rafael Rosell ${ }^{1,3,6,7} \&$ Miguel-Angel Molina-Vila ${ }^{1}$

Non-small cell lung cancer (NSCLC) tumors harboring mutations in EGFR ultimately relapse to therapy with EGFR tyrosine kinase inhibitors (EGFR TKIs). Here, we show that resistant cells without the p.T790M or other acquired mutations are sensitive to the Aurora B (AURKB) inhibitors barasertib and S49076. Phospho-histone $\mathrm{H3}(\mathrm{pH} 3)$, a major product of $A U R K B$, is increased in most resistant cells and treatment with AURKB inhibitors reduces the levels of $\mathrm{pH} 3$, triggering $\mathrm{G} 1 / \mathrm{S}$ arrest and polyploidy. Senescence is subsequently induced in cells with acquired mutations while, in their absence, polyploidy is followed by cell death. Finally, in NSCLC patients, pH3 levels are increased after progression on EGFR TKIs and high $\mathrm{pH} 3$ baseline correlates with shorter survival. Our results reveal that AURKB activation is associated with acquired resistance to EGFR TKIs, and that AURKB constitutes a potential target in NSCLC progressing to anti-EGFR therapy and not carrying resistance mutations.

\footnotetext{
${ }^{1}$ Laboratory of Oncology, Pangaea Oncology, Quiron Dexeus University Hospital, 08028 Barcelona, Spain. ${ }^{2}$ Institut de Recherches Internationales Servier, 92284 Suresnes, France. ${ }^{3}$ Instituto Oncológico Dr. Rosell, Quiron-Dexeus University Hospital, 08028 Barcelona, Spain. ${ }^{4}$ Clinical and Translational Oncology Group, Institute of Oncology, Fundacion Santa Fe de Bogotá, Bogotá 110111, Colombia. ${ }^{5}$ Foundation for Clinical and Applied Cancer Research (FICMAC), Bogotá 110111, Colombia. ${ }^{6}$ Catalan Institute of Oncology, Hospital Germans Trias i Pujol, 08916 Badalona, Spain. ${ }^{7}$ Germans Trias i Pujol, Health Sciences Institute and Hospital, Campus Can Ruti, 08916 Badalona, Spain. ${ }^{8}$ Present address: Servicio Anatomía Patológica, Hospital Clínic de Barcelona, Barcelona 08036, Spain. Correspondence and requests for materials should be addressed to M.-A.M-V. (email: mamolina@panoncology.com)
} 
ung tumors are the leading cause of cancer mortality worldwide ${ }^{1}$, being non-small cell lung cancer (NSCLC) the most common subtype. Activating mutations in the Epidermal Growth Factor Receptor (EGFR) gene are present in $14-17 \%$ of advanced NSCLC in European populations ${ }^{2}$ and $40-50 \%$ in East $\mathrm{Asia}^{3}$. Tumors with the most common alterations, exon 19 deletions and exon 21 point mutations (p.L858R), are sensitive to EGFR tyrosine kinase inhibitors (EGFR TKIs) such as gefitinib ${ }^{4}$, erlotinib ${ }^{5}$, or afatinib $^{6}$. However, treated patients ultimately develop resistance, leading to disease progression 5 .

The most frequent mechanism associated with acquisition of resistance is the p.T790M mutation in $E G F R^{7}$, which emerges in $40-60 \%$ of tumors progressing on first and second generation EGFR TKIs. Additional mechanisms include amplification, overexpression and/or autocrine activation loops involving membrane receptors such as MET proto-oncogene (MET), Fibroblast Growth Factor Receptor (FGFR) or AXL receptor tyrosine kinase $(\mathrm{AXL})^{8-11}$. Finally, activating mutations of $B R A F$ and PIK3CA, amplification of MAPK1, gene fusions involving FGFR3, histological transformation, and epithelial-tomesenchymal transition (EMT) have also been associated with progression to EGFR TKIs ${ }^{8}$.

The characterization of the mechanisms responsible for acquired resistance to EGFR TKIs is of both fundamental and clinical interest and drugs that target some of the proteins mentioned above are currently in clinical trials. Regarding the $\mathrm{p}$. T790M, third generation EGFR TKIs are active against it and one of them, osimertinib, has been approved for second line treatment ${ }^{12}$. However, similarly to gefitinib, erlotinib or afatinib, patients ultimately progress. Mechanisms of resistance to third generation EGFR TKIs include loss of the p.T790M mutation ${ }^{13}$, emergence of a further mutation in exon 20 of EGFR (p.C797S) ${ }^{14}$, MET and HER2 activation, and de novo mutations in $K R A S^{15}$.

In order to gain insight into the mechanisms associated with acquired resistance in EGFR-mut NSCLC, we have generated cell lines resistant to EGFR TKIs. Unexpectedly barasertib, an Aurora $\mathrm{B}$ (AURKB) inhibitor ${ }^{16,17}$, shows antitumor activity in resistant cells lacking the p.T790M or other acquired mutations. AURKB is a ubiquitously expressed serine/threonine kinase that phosphorylates histone $\mathrm{H} 3$ on Ser10 and variant centrosome protein A on Ser7 in early G2, leading to condensation of chromatin ${ }^{18}$. Later, it regulates the spindle assembly complex, and inhibition or loss of AURKB leads to defective chromosome segregation and polyploidy ${ }^{19}$. Amplification or overexpression of AURKB has been associated with poor prognosis in several human tumors and AURKB inhibitors are in phase I-II clinical trials for leukemia ${ }^{18,20}$. AURKB has also been implicated in resistance to certain antitumor agents, such as aromatase inhibitors in breast carcinoma $^{21}$, paclitaxel in NSCLC ${ }^{22}$, cetuximab in head and neck squamous cell carcinoma ${ }^{23}$, or vemurafenib in melanoma ${ }^{24}$. However, no role has been reported for AURKB in the context of resistance to targeted therapies in NSCLC.

Our results indicate that AURKB is activated in NSCLC tumor cells with acquired resistance to EGFR TKIs and can be a therapeutic target in absence of resistance mutations. Clinical trials are thus warranted to determine the efficacy of multi-targeted agents inhibiting not only RTKs, but also AURKB, in EGFRmutant patients progressing on EGFR TKIs and not harboring acquired mutations.

\section{Results}

Resistance to EGFR TKIs in PC9 cells is associated with different alterations. We had previously generated six EGFR-TKIresistant cell lines by treating EGFR-mut, TKI-sensitive PC9 cells with increasing concentrations of gefitinib (PC9-GR1-5) or erlotinib (PC9-ER). While all six of them retained the 15-bp deletion in the EGFR gene present in the parental PC9, the p.T790M mutation only emerged in PC9-GR1 and GR4 ${ }^{25}$. Both cell lines were sensitive to osimertinib (Table 1). Subsequently, we generated 17 additional lines resistant to osimertinib by treating PC9-GR1 and GR4 with increasing concentrations of the drug; eight of them lost the p.T790M mutation and five also the exon 19 deletion. The p.C797S mutation did not emerge in any case. Six of the osimertinib-resistant cell lines were selected for further work, together with the six lines resistant to first generation EGFR TKIs (Fig. 1a and Table 1). Next generation sequencing (NGS) did not reveal other acquired mutations in BRAF, KRAS, NRAS, or EGFR. The PC9-GR1 and GR4 cell lines, harboring the p. T790M mutation at 25 and $38 \%$ allelic fractions, were similar to the parental PC9 under microscopic examination; the rest of the lines showed a more mesenchymal phenotype (Supplementary Fig. 1a).

Western blotting, immunohistochemistry (IHC) and mRNA analyses revealed overexpression of AXL in 10 of the resistant cell lines, MET activation in three and FGFR1 upregulation in two (Figs. 1b-c, Table 1 and Supplementary Fig. 1b). HER2 and MET were not amplified by FISH or NGS in any case. Molecular alterations frequently co-occurred (Table 1). Interestingly, GAS6 expression was significantly elevated in all the resistant cells, particularly in those with AXL upregulation (Fig. 1d and Supplementary Fig. 1c).

Resistant cells are insensitive to AXI, MET, or FGFR1 inhibition. Next, we used viability assays to determine the sensitivity of the PC9-derived cell lines to several targeted agents (Table 1). As expected, p.T790M-negative cells resistant to first generation EGFR TKIs (PC9-GR2, GR3, GR5, and ER) were insensitive to afatinib and osimertinib, in contrast to the $p$. T790M-positive cells (PC9-GR1 and GR4). The osimertinibresistant lines derived from PC9-GR1 and GR4 also acquired resistance to afatinib and remained insensitive to first generation EGFR TKIs.

The resistant cell lines with AXL upregulation had IC50s around $2-3 \mu \mathrm{M}$ for the AXL inhibitor BGB324, indistinguishable from the parental PC9 or from the resistant cells not overexpressing AXL. A similar behavior was observed in the case of the MET inhibitors capmatinib and crizotinib, where the IC50s did not correlate with MET activation. Resistant cells also remained largely insensitive to the combination of BGB324 with capmatinib (Supplementary Fig. 2). The FGFR1 over-expressing PC9-GR5 cells showed an IC50 of $2.3 \mu \mathrm{M}$ for the FGFR1 inhibitor nintedanib; only 2-10 times lower than the rest of the panel.

Western blotting showed that crizotinib at $2 \mu \mathrm{M}$ effectively suppressed the phosphorylation of MET in PC9-GR1, while BGB324 at the same concentration inhibited the activation of AXL in PC9-ER, and nintedanib the phosphorylation of FGFR substrate 2 (FRS2), the main downstream effector of FGFR1, in PC9-GR5. These results demonstrated that these TKIs, despite showing limited antiproliferative activity in the resistant cells, were able to block their RTK targets at the concentrations used in the MTT assays (Supplementary Fig. 3a).

Finally, since upregulation of AXL was common in our panel of resistant cell lines, we silenced $A X L$ expression in two non-p. T790M cells, PC9-ER, and PC9-GR3. The AXL-silenced clones did not show any morphological changes and had growth curves indistinguishable from the control clones. In addition, no significant differences were found in the sensitivity to any of the drugs tested, including gefitinib (Supplementary Table 1 and Supplementary Fig. 3b-d). 
Table 1 Characteristics (left) and IC50s ( $\mu \mathrm{M}$, right) for selected drugs of the PC9-derived cell lines used in the study

\begin{tabular}{|c|c|c|c|c|c|c|c|c|c|c|c|c|c|c|c|c|}
\hline Cell line & $\begin{array}{l}\text { Doublintg } \\
\text { time (h) }\end{array}$ & $\begin{array}{l}\text { EGFR } \\
\text { Del19 }\end{array}$ & $\begin{array}{l}\text { EGFR } \\
790 \mathrm{M}\end{array}$ & $\begin{array}{l}\text { AXL over- } \\
\text { expression }\end{array}$ & $\begin{array}{l}\text { MET } \\
\text { activation }\end{array}$ & $\begin{array}{l}\text { FGFR1 over- } \\
\text { expression }\end{array}$ & $\begin{array}{l}\text { Gefitinib } \\
\text { (EGFR) }\end{array}$ & $\begin{array}{l}\text { Erlotinib } \\
\text { (EGFR) }\end{array}$ & $\begin{array}{l}\text { Afatinib } \\
\text { (EGFR) }\end{array}$ & $\begin{array}{l}\text { Osimertinib } \\
\text { (EGFR) }\end{array}$ & $\begin{array}{l}\text { S49076 } \\
\text { (AXL, } \\
\text { MET, } \\
\text { FGFR1, } \\
\text { AURKB) } \\
\end{array}$ & $\begin{array}{l}\text { Foretinib } \\
\text { (MET, } \\
\text { KDR, } \\
\text { AXL, } \\
\text { AURKB) }\end{array}$ & $\begin{array}{l}\text { Crizotinib } \\
\text { (MET, } \\
\text { ALK) } \\
\end{array}$ & $\begin{array}{l}\text { Capmatinib } \\
\text { (MET) }\end{array}$ & $\begin{array}{l}\text { BGB324 } \\
\text { (AXL) }\end{array}$ & $\begin{array}{l}\text { Nintedanib } \\
\text { (FGFR) }\end{array}$ \\
\hline PC9 & $24 \pm 2$ & + & $v-$ & - & - & - & 0.04 & 0.005 & 0.003 & 0.05 & $>50$ & 1.6 & 2.0 & $>25$ & 2.3 & 11.4 \\
\hline $\begin{array}{l}\text { PC9-GR1 } \\
\text { PC9-GR2 } \\
\text { PC9-GR3 } \\
\text { PC9-GR4 } \\
\text { PC9-GR5 } \\
\text { PC9-ER }\end{array}$ & $\begin{array}{l}27 \pm 3 \\
31 \pm 1 \\
30 \pm 1 \\
29 \pm 1 \\
31 \pm 5 \\
27 \pm 2\end{array}$ & $\begin{array}{l}+ \\
+ \\
+ \\
+ \\
+ \\
+\end{array}$ & $\begin{array}{l}+ \\
- \\
- \\
+ \\
- \\
-\end{array}$ & $\begin{array}{l}- \\
+ \\
+ \\
+ \\
++ \\
++\end{array}$ & $\begin{array}{l}+ \\
+ \\
- \\
- \\
- \\
-\end{array}$ & $\begin{array}{l}- \\
- \\
- \\
- \\
++ \\
-\end{array}$ & $\begin{array}{l}12.2 \\
14.9 \\
15.8 \\
6.5 \\
18.1 \\
12.2 \\
\end{array}$ & $\begin{array}{l}4.8 \\
25.0 \\
33.7 \\
3.8 \\
21.8 \\
28.6 \\
\end{array}$ & $\begin{array}{l}0.4 \\
3.8 \\
4.8 \\
0.4 \\
3.6 \\
6.8\end{array}$ & $\begin{array}{l}0.03 \\
3.0 \\
5.5 \\
0.02 \\
5.3 \\
3.2 \\
\end{array}$ & $\begin{array}{l}50 \\
1.2 \\
0.3 \\
>50 \\
0.9 \\
0.3 \\
\end{array}$ & $\begin{array}{l}1.5 \\
0.6 \\
0.5 \\
3.6 \\
0.6 \\
0.7\end{array}$ & $\begin{array}{l}2.4 \\
2.3 \\
1.0 \\
2.1 \\
2.1 \\
1.4 \\
\end{array}$ & $\begin{array}{l}>25 \\
>25 \\
>25 \\
>25 \\
19.8 \\
>25\end{array}$ & $\begin{array}{l}2.3 \\
2.5 \\
2.7 \\
3.3 \\
2.7 \\
3.3 \\
\end{array}$ & \begin{tabular}{l|}
14.3 \\
5.7 \\
8.1 \\
12.5 \\
2.3 \\
7.8 \\
\end{tabular} \\
\hline $\begin{array}{l}\text { PC9-GR1-AZD1 } \\
\text { PC9-GR1-AZD2 } \\
\text { PC9-GR1-AZD3 } \\
\text { PC9-GR1-AZD4 } \\
\text { PC9-GR4-AZD1 } \\
\text { PC9-GR4-AZD2 }\end{array}$ & $\begin{array}{l}32 \pm 5 \\
25 \pm 4 \\
26 \pm 6 \\
30 \pm 3 \\
26 \pm 4 \\
34 \pm 7\end{array}$ & $\begin{array}{l}- \\
- \\
+ \\
- \\
+ \\
+\end{array}$ & $\begin{array}{l}- \\
- \\
- \\
- \\
+ \\
+^{a}\end{array}$ & $\begin{array}{l}++ \\
++ \\
+ \\
+ \\
+ \\
-\end{array}$ & $\begin{array}{l}- \\
- \\
+ \\
- \\
- \\
-\end{array}$ & $\begin{array}{l}- \\
- \\
- \\
- \\
- \\
+\end{array}$ & $\begin{array}{l}21.0 \\
20.0 \\
18.7 \\
18.2 \\
10.5 \\
13.7\end{array}$ & $\begin{array}{l}43.9 \\
49.8 \\
42.4 \\
35.5 \\
43.2 \\
41.2 \\
\end{array}$ & $\begin{array}{l}15.3 \\
17.0 \\
15.4 \\
13.5 \\
7.1 \\
2.6 \\
\end{array}$ & $\begin{array}{l}8.3 \\
8.8 \\
5.7 \\
6.4 \\
5.1 \\
7.5 \\
\end{array}$ & $\begin{array}{l}0.2 \\
0.3 \\
0.2 \\
0.3 \\
>50 \\
0.4\end{array}$ & $\begin{array}{l}0.6 \\
0.7 \\
0.6 \\
0.7 \\
2.5 \\
4.2\end{array}$ & $\begin{array}{l}0.7 \\
1.3 \\
0.6 \\
0.7 \\
1.9 \\
5.8\end{array}$ & $\begin{array}{l}>25 \\
>25 \\
>25 \\
>25 \\
21.9 \\
>25\end{array}$ & $\begin{array}{l}2.1 \\
2.4 \\
2.1 \\
2.1 \\
2.6 \\
2.3\end{array}$ & $\begin{array}{l}24.2 \\
13.3 \\
24.9 \\
14.3 \\
13.7 \\
22.2\end{array}$ \\
\hline $\mathrm{H} 1975$ & $21 \pm 1$ & - & + & - & nd & - & 9.1 & 24.5 & 0.0005 & 0.23 & 22.1 & 2.5 & 1.5 & nd & 1.8 & nd \\
\hline
\end{tabular}

p.T790M-negative cells are sensitive to AURKB inhibition. Of all the TKIs tested foretinib, a type II inhibitor that targets MET, AXL, and other RTKs but also AURKB ${ }^{26}$, showed the lowest IC50 in most of the resistant cell lines, particularly in those p.T790Mnegative (Table 1). This result prompted us to test other AURKB inhibitors. We found that barasertib, a drug specifically targeting AURKB, had a strong antiproliferative effect in MTT assays in p. T790M-negative cells, with IC50s $<0.06 \mu \mathrm{M}$ in contrast to $>10$ $\mu \mathrm{M}$ in the p.T790M-positive and the parental PC9 cells (Table 2, Fig. 1e, Supplementary Fig. 4). The only exception was PC9-GR2, a p.T790M-negative cell line with MET activation, where barasertib showed little effect. The AURKA/AURKB inhibitor tozasertib was also very active against non-p.T790M cells, although the differences in the IC50s were less pronounced than in the case of barasertib. In view of these results, we included in our study the H1975 NSCLC cell line, a model of intrinsic resistance to EGFR TKIs that harbors a p.L858R and a p.T790M mutation in EGFR at $75 \%$ allelic fraction (Table 1). Similarly to the p.T790M-positive PC9-derived cells, H1975 was found to be resistant to barasertib and tozasertib (Table 2, Fig. 1e).

Cells treated with barasertib, particularly those p.T790Mpositive, appeared significantly enlarged, vacuolized and often multinucleated under microscopic examination (Supplementary Fig. 5a). In consequence, growth curves were determined for PC9-ER (p.T790M negative) and PC9-GR4 (p.T790M positive) cells comparing direct counting vs. viability assays (MTT). Remarkable differences were observed in the case of the PC9GR4, with a significant dose-dependent effect of AURKB inhibition on cell numbers, which was not reflected in total cell metabolism, as estimated by MTT, probably as a result of the considerably larger size of the cells (Supplementary Fig. 5b-c).

S49076 is active against p.T790M-negative resistant cells. Next, we tested the effects of S49076, another type II inhibitor, in our panel of resistant cells. S49076 targets MET, AXL, and FGFR1/2/ $3^{27}$ but also shows a significant in vitro activity against AURKB, with an IC50 of $3 \mathrm{nM}$ on the isolated enzyme ${ }^{28}$. Similarly to barasertib or tozasertib, parental PC9 and the PC9-derived cell lines carrying the p.T790M were resistant to the S49076, with IC50s over $50 \mu \mathrm{M}$, while the p.T790M-negative cell lines were sensitive to the drug (Supplementary Fig. 6a). Interestingly, the PC9-GR4-AZD2 cells, which harbor the p.T790M mutation at very low allelic fractions, showed an intermediate behavior. Although barasertib had lower IC50s than S49076 in most of the p.T790M-negative cells, surviving populations were often observed at $\geq 1 \mu \mathrm{M}$ concentrations of barasertib but not of $\mathrm{S} 49076$ (Table 2).

S49076 was able to block the phosphorylation of its target RTKs at concentrations within the range used in the MTT assays (Supplementary Fig. 3a). However, several lines of evidence indicated that inhibition of RTKs was insufficient to explain the antitumor effects of S49076 and that AURKB inhibition was playing a significant role. First, S49076 showed significantly stronger antiproliferative activity than the combination of an AXL and a MET inhibitor (Supplementary Fig. 2). Second, the $A X L$-silenced cells showed IC50s for S49076 indistinguishable from the parental or control cells (Supplementary Table 1 and Supplementary Fig. 3d). Third, western blotting revealed that S49076 induced only a moderate reduction in the levels of pAKT and/or pERK1/2 in the non-p.T790M-resistant cells (Supplementary Fig. 6b-c). Fourth, non-p.T790M-resistant cells treated with S49076 showed morphological changes similar to those observed in the case of barasertib and remarkable differences between direct counting vs. viability assays (MTT) (Supplementary Fig. 7).

pH3 but not AURKB is elevated in resistant cells. The previous results suggested that AURKB could be a target in EGFR-TKIresistant cells negative for the p.T790M mutation. A series of experiments were performed to test this hypothesis. First, we determined the levels of total AURKB by mRNA expression analyses, Western blotting and immunofluorescent staining, and found no significant differences between the parental and the resistant PC9 cells (Figs. 2a-c). In contrast, a significant increase in $\mathrm{pH} 3$, the main product of AURKB activity, was observed in eight of the 12 EGFR-TKI-resistant cells, both p.T790M-positive and negative (Figs. 2d-e). During cell cycle progression, histone $\mathrm{H} 3$ is phosphorylated in early G2 and binds to mitotic chromosomes. However, the total increase of $\mathrm{pH} 3$ levels in the resistant cell lines did not associate with a higher percentage of proliferating cells, as revealed by Ki67 IHC (Figs. 2f-g).

IHC also showed that the $\mathrm{pH} 3$ positivity in the parental PC9 line was mainly circumscribed to mitotic nuclei or dividing cells, which showed an intense staining $(3+)$. In contrast, in the case of the EGFR-TKI-resistant cells, a significant number of apparently resting cells also showed a weak or moderate nuclear staining $(1+$ or $2+)$. Interestingly, the $\mathrm{H}$-score for mitotic $(3+) \mathrm{pH} 3$ closely correlated with Ki67 immunostaining in our panel of resistant lines, but the $\mathrm{H}$-score corresponding to apparently resting cells $(1+$ or $2+$ ) did not (Fig. $2 \mathrm{~g}$ ). These results were coincident with immunofluoresence studies, where $\mathrm{pH} 3$ staining 
a

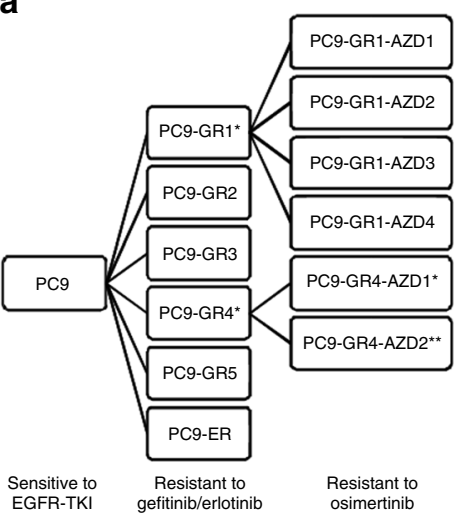

b

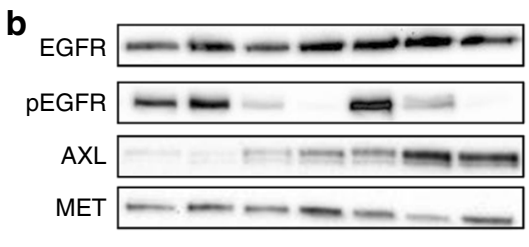

PMET

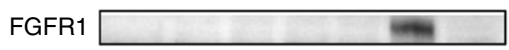

pFRS $\square$

$\beta$-Tubulin

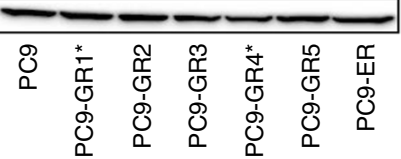

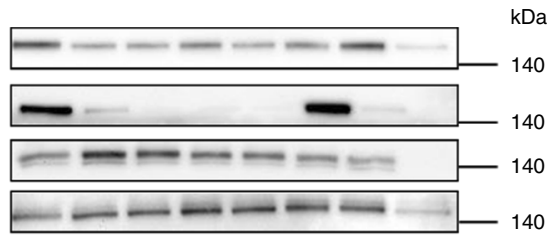

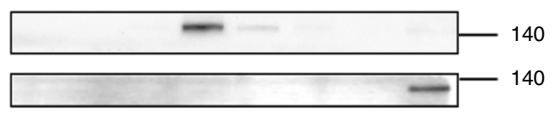

C

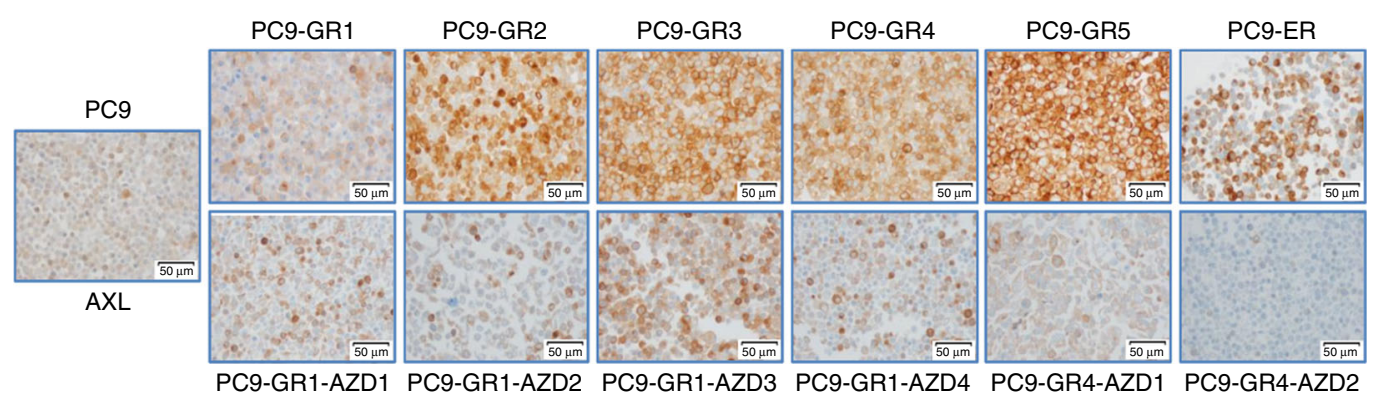

d

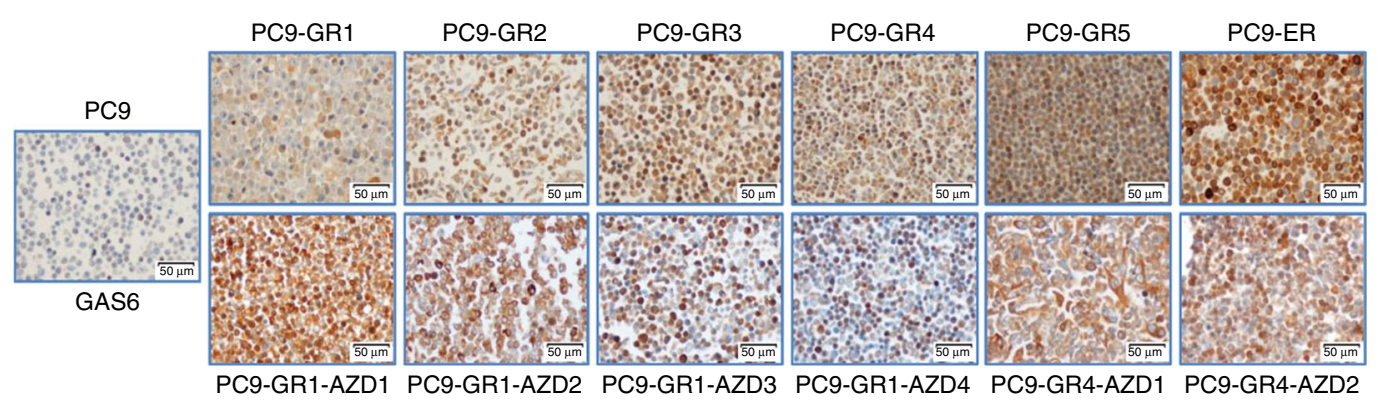

e
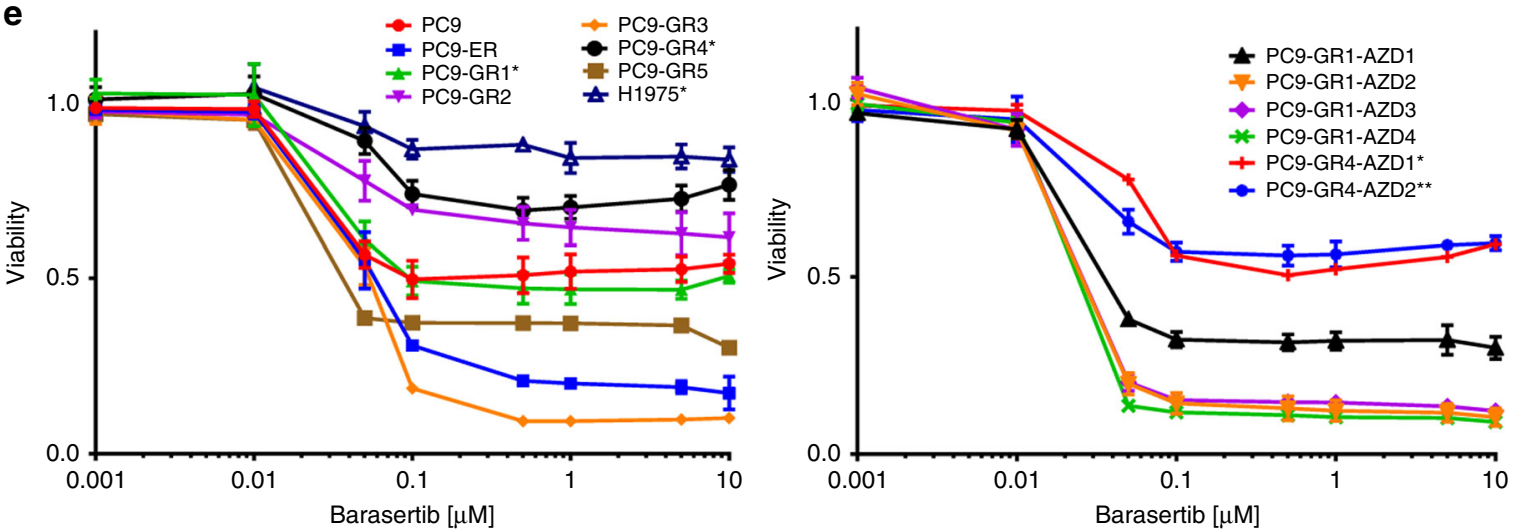

Fig. 1 Characterization of a panel of PC9-derived cell lines resistant to EGFR TKIs. a Diagram summarizing the establishment of the panel. Asterisks indicate cell lines harboring the p.T790M-resistant mutation at $>0.1 \%\left(^{\star}\right)$ or $<0.1 \%\left(^{\star \star}\right)$ allelic fraction. $\mathbf{b}$ Western blot analysis of proteins and phospho-proteins related with the emergence of resistance. pFRS, phospho-FGFR substrate 2. c IHC of AXL. d IHC of GAS6. Scale bars indicate $50 \mu$ m. e Dose-response curves of the parental, the EGFR-TKI-resistant and the H1975 cells to the AURKB inhibitor barasertib at $72 \mathrm{~h}$. Values shown are means \pm standard deviation $(\mathrm{SD})$, experiments were conducted in tri $(n=3)$ or quadruplicates $(n=4)$. In each experiment, every concentration of drug was tested in sextuplicates $(n=6)$ 
Table 2 Sensitivity to Aurora inhibitors of the PC9-derived cell lines used in the study

\begin{tabular}{|c|c|c|c|c|c|c|}
\hline Cell line ${ }^{a}$ & $\begin{array}{l}\text { Barasertib } \\
\text { (AURKB) } \\
\text { IC50 ( } \mu M)\end{array}$ & $\begin{array}{l}\text { Barasertib } \\
\text { (AURKB) } \\
\% \text { Cells }\end{array}$ & $\begin{array}{l}\text { Tozasertib } \\
\text { (AURKA, AURKB) } \\
\text { IC50 ( } \mu \mathrm{M})\end{array}$ & $\begin{array}{l}\text { Tozasertib } \\
\text { (AURKA, AURKB) } \\
\% \text { Cells }\end{array}$ & $\begin{array}{l}\text { S49076 (AURKB, } \\
\text { AXL, MET, FGFR1) } \\
\text { IC50 ( } \mu \mathrm{M})\end{array}$ & $\begin{array}{l}\text { S49076 (AURKB, } \\
\text { AXL, MET, FGFR1) } \\
\% \text { Cells }\end{array}$ \\
\hline PC9 & 12.7 & 52 & 0.7 & 44 & $>50$ & 55 \\
\hline PC9-GR1 & 13.9 & 45 & 5.7 & 65 & $>50$ & 56 \\
\hline PC9-GR2 & 30.7 & 69 & 0.5 & 47 & 1.2 & 54 \\
\hline PC9-GR3 & 0.05 & 9 & 0.1 & 14 & 0.3 & 4 \\
\hline PC9-GR4 & 34.4 & 67 & 7.8 & 63 & $>50$ & 65 \\
\hline PC9-GR5 & 0.04 & 37 & 0.2 & 25 & 0.9 & 33 \\
\hline PC9-ER & 0.06 & 20 & 0.2 & 35 & 0.3 & 3 \\
\hline PC9-GR1-AZD1 & 0.04 & 32 & 0.2 & 37 & 0.2 & 9 \\
\hline PC9-GR1-AZD2 & 0.03 & 12 & 0.05 & 14 & 0.3 & 8 \\
\hline PC9-GR1-AZD3 & 0.03 & 15 & 0.08 & 26 & 0.2 & 6 \\
\hline PC9-GR1-AZD4 & 0.03 & 10 & 0.1 & 16 & 0.3 & 7 \\
\hline PC9-GR4-AZD1 & 27.5 & 64 & 2.6 & 53 & $>50$ & 57 \\
\hline PC9-GR4-AZD2 & 23.2 & 55 & 4.6 & 50 & 0.4 & 31 \\
\hline $\mathrm{H} 1975$ & $>50$ & 83 & 28.8 & 84 & 22.1 & 76 \\
\hline
\end{tabular}

in resistant cells was present in dividing but also in apparently resting nuclei, often concentrated in foci (Fig. 3).

Western blotting and immunofluorescence demonstrated a dose-dependent reduction in $\mathrm{pH} 3$ by barasertib at concentrations $\geq 0.05 \mu \mathrm{M}$ in the p.T790M-negative PC9-ER and the p.T790Mpositive PC9-GR4 cells. Similar effects were observed in the case of S49076 at $\geq 0.1 \mu \mathrm{M}$ (Figs. 3a-b, Supplementary Fig. 6d-e). The reduction in $\mathrm{pH} 3$ levels was primarily observed in apparently resting cells, with weakening or disappearance of foci, while the strong $\mathrm{pH} 3$ staining in mitotic cells was not altered (Fig. 3b).

AURKB silencing de-sensitizes non-p.T790M cells to AURKB inhibition. Next, we transfected PC9-ER cells with shRNA lentivirus particles to silence $A U R K B$. The number of puromycinresistant clones was low and they took several weeks to emerge. Western blotting, immunofluorescence and gene expression analyses confirmed partial, but not complete, silencing of $A U R K B$ (Figs. 3c-d). Although the doubling time of the clones with $A U R K B$ silencing and the parental PC9-ER cells was not significantly different (Supplementary Table 1), microscopic observation revealed profound changes in the morphology of the partly silenced cells, which uniformly showed an enlarged cytoplasm, aberrant mitotic figures and a significant fraction of polynucleated cells (Supplementary Fig. 8). Finally, immunofluorescence demonstrated reduced levels of $\mathrm{pH} 3$ in apparently resting but not in dividing cells associated with $A U R K B$ partial silencing (Fig. 3d). These changes in $\mathrm{pH} 3$ staining strongly resembled those observed when treating parental PC9-ER and PC9-GR4 cells with AURKB inhibitors.

Then, we examined the sensitivity to several drugs of the PC9ER clones partially silenced for AURKB. No significant differences were observed in the case of gefitinib, BGB324 or other TKIs. In contrast, the partly silenced clones showed a $>100$-fold increase in the IC50s for barasertib and S49076, compared to parental or control-transfected PC9-ER cells (Fig. $3 \mathrm{e}$ and Supplementary Table 1), confirming that AURKB is the main target of both drugs in PC9-ER. This result was further validated using CRISPR/ Cas9 system to knockout AURKB from PC9-ER. Similarly to the results obtained when using shRNA, we could only isolate clones with partial silencing (Supplementary Fig. 8b). Also, the AURKB silenced clones showed a $>10$-fold increase in the IC50 for barasertib (Supplementary Fig. 8c), while the IC50s for gefitinib remained unchanged.
S49076 has antitumor activity on PC9-ER xenografts. Animal experiments revealed that S49076 inhibited the growth of PC9-ER xenografts in a dose-dependent way. At the end of the 3-week treatment, there was a $55 \%$ reduction in tumor growth at the highest dosage of $37.5 \mathrm{mg} / \mathrm{kg} /$ day. However, this inhibition did not reach statistical significance, probably due to the high variability in the size of the xenografts and the relatively small number of animals in each group. PC9-GR4 xenografts were found to be less sensitive to $\$ 49076$, with a reduction of only $21 \%$ in tumor growth at $37.5 \mathrm{mg} / \mathrm{kg} /$ day (Fig. 3f and Supplementary Fig. 9).

At sacrifice, subcutaneous tumors from PC9-ER were included in paraffin. IHC revealed that the total levels of $\mathrm{pH} 3$ in the tumors from S49076-treated mice decreased in a dose-dependent way, while the levels of Ki67 did not. Similarly to cultured PC9-ER, the $\mathrm{pH} 3$ staining of xenografts revealed intense staining in mitotic cells and moderate or weak staining in a number of apparently resting cells. The inhibitory effect of S49076 on pH3 levels was found to be circumscribed to the latter (Figs. $3 g-h$ ).

AURKB inhibition induces cell death or senescence. Inhibition of AURKB has been described to trigger cell cycle arrest and polyploidy. Flow cytometry revealed that barasertib and S49076 at concentrations corresponding to the IC50s induced a significant increase in the fraction of G2/M cells after $24 \mathrm{~h}$ in the p.T790Mpositive PC9-GR4 and the p.T790M-negative PC9-ER. This effect was accompanied by the appearance of a significant fraction of polyploid cells, particularly in the case of PC9-GR4 (Fig. 4a).

Proliferation assays had revealed that barasertib and S49076 had a strong antiproliferative activity in non-p.T790M-resistant cells. Consequently, we investigated if these drugs could induce cell death following cycle arrest. Flow cytometry experiments after Annexin V and PI staining showed that AURKB inhibition triggered apoptosis/necrosis after $24 \mathrm{~h}$ in all the p.T790Mnegative cell lines tested, namely PC9-ER, PC9-GR3, and PC9GR1AZD3. In contrast, no cell death was observed in the $p$. T790M-positive cells PC9-GR4, PC9-GR1, PC9-GR4AZD1, and H1975 (Figs. 4b-f). Western blotting for cleaved PARP detection confirmed this result (Fig. 4c). In the case of the p.T790Mpositive cells, microscopic observation revealed that polyploid cells could survive several days in presence of the drugs.

Following polyploidy, AURKB inhibitors induce senescence in several cell types, via the ATM/Chk2 DNA damage response (DDR). Senescent cells are characterized by permanent growth 
a

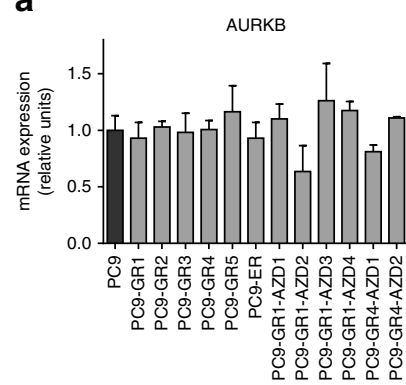

b

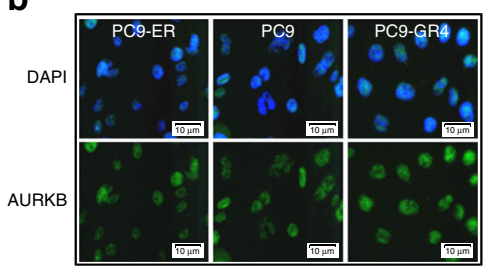

C

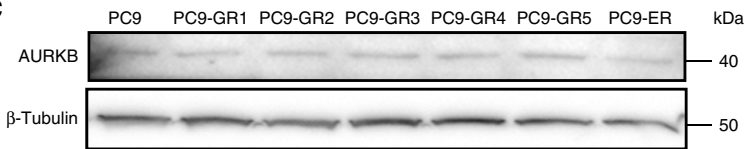

d

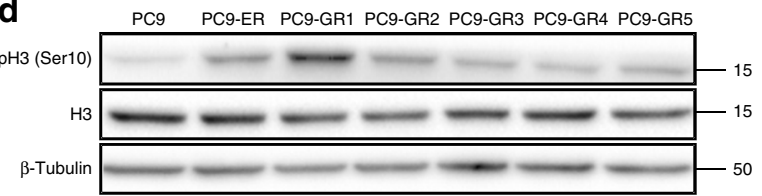

e

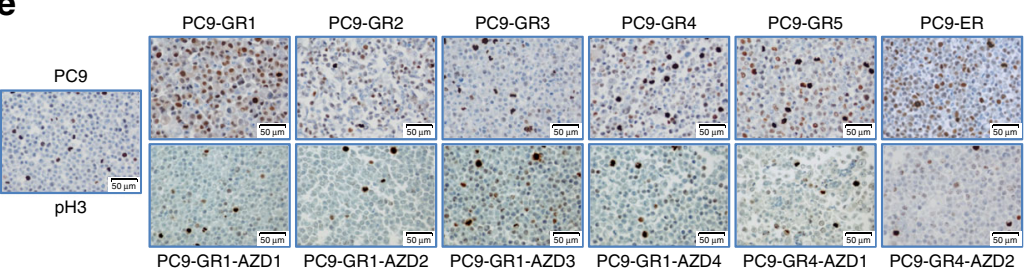

f
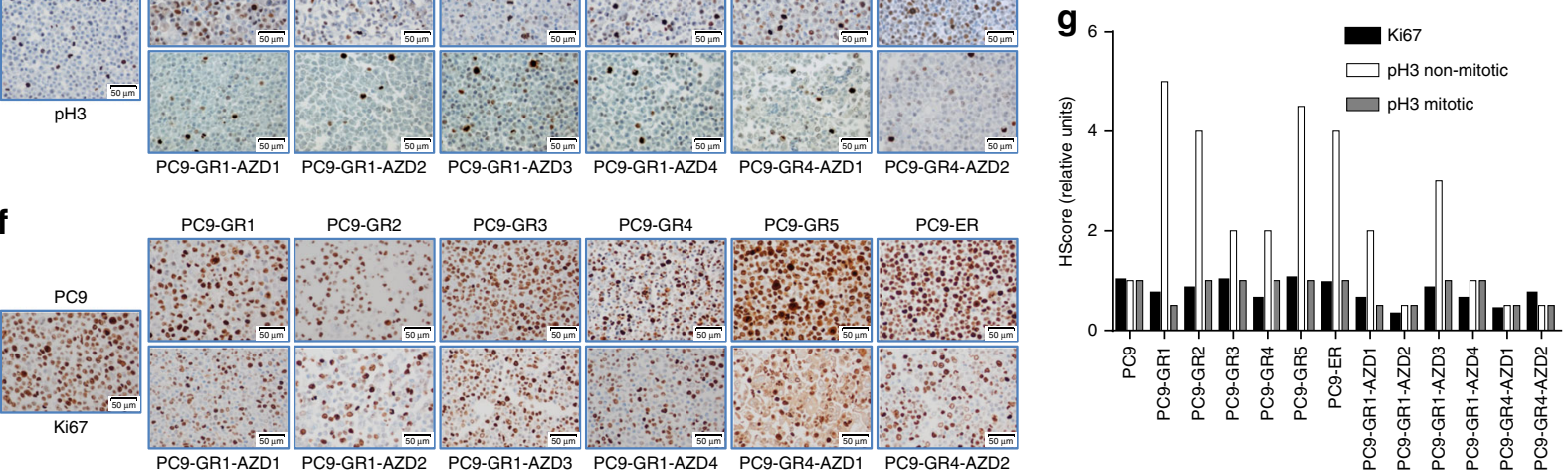

Fig. 2 AURKB and pH3 in PC9-derived cell lines resistant to EGFR TKIs. a Levels of AURKB mRNA in the cells of the panel. Values shown are means \pm SD of three independent determinations $(n=3)$. $\mathbf{b}$ Levels of AURKB by ICC in the parental PC9 and the resistant lines PC9-ER (p.T790M negative) and PC9-GR4 (p.T790M positive). Scale bars indicate $10 \mu \mathrm{m}$. c Levels of AURKB in the parental PC9 and the cell lines resistant to first generation EGFR TKIs by western blotting. $\mathbf{d}$ Levels of histone $\mathrm{H} 3$ and pH3 in the same cell lines by western blotting. e-g IHC of pH3 (e), Ki67 (f) and $\mathrm{H}$-score of Ki67, mitotic pH3 and nonmitotic $\mathrm{pH} 3(\mathbf{g})$ in the 12 cell lines resistant to EGFR TKIs. Scale bars indicate $50 \mu \mathrm{m}$. Values shown are means of two blind determinations performed independently by two expert pathologists $(n=2)$

arrest, enlarged nucleus and cellular size, vacuolization, overexpression of acidic beta-galactosidase and the senescenceassociated secretory phenotype (SASP), mediated by NF- $\kappa \mathrm{B}$ and characterized by the secretion of growth factors (i.e., HGF) and other proteins ${ }^{29}$. We used several techniques to determine if AURKB inhibition induced senescence in our resistant cell lines. Beta-galactosidase staining revealed a significant number of positive cells after $48 \mathrm{~h}$ treatment with barasertib in all the p. T790M-positive cell lines tested, that included PC9-GR1, PC9-GR4, PC9-GR4AZD1, and H1975 (Figs. 4d-g). Remarkably, cells over-expressing acidic beta-galactosidase were almost invariably polyploid under microscopic examination. Regarding the p.T790Mnegative cells, no senescence was apparent in the PC9-GR3 while a weak positivity associated with polyploidy in the few cells surviving at the highest concentration of drug $(0.1 \mu \mathrm{M})$ was observed in the case of PC9-ER and PC9-GR1-AZD3. Similar results were obtained for S49076 in the PC9-GR4 cells, while no beta-galactosidase positivity was apparent in the PC9-ER (Fig. 4d). Next, we used C12FDG followed by flow cytometry to quantify the effect, and we found a dose-dependent increase in the percentage of betagalactosidase-positive cells in PC9-GR4 cells, which reached 65\% at $1 \mu \mathrm{M}$ S49076. In contrast, no positivity was observed in PC9-ER (Fig. 4e). Finally, mRNA analyses showed up-regulation of the DDR genes ATM, CHK-2, and 53BP1, together with $N F-\kappa B$, in the PC9GR4 but not in the PC9-ER cells treated with barasertib or S49076 (Fig. 4h). Taken together, these findings confirmed the induction of DDR-associated senescence and NF- $\mathrm{BB}$-mediated SASP in the p.T790M-positive resistant cells.

Senescence is a heterogeneous phenotype, and several examples have been described of pseudo-senescent cells where growth arrest was reversible ${ }^{29}$. In order to determine if this was also the case in the PC-GR4 cells, barasertib $(0.01-0.2 \mu \mathrm{M})$ or S49076 $(0.1-1 \mu \mathrm{M})$ were removed after 2, 3 and 6 days. Growth arrest and morphological changes were found to be permanent after 2 days in cells treated with $0.2 \mu \mathrm{M}$ barasertib and $1 \mu \mathrm{M}$ S49076, while lower concentrations required incubation times up to 6 days.

11-18-derived resistant cells are sensitive to AURKB inhibition. In order to validate our findings in an additional model of resistance to EGFR TKIs, we used six p.T790M-negative gefitinibresistant lines (GR1-GR6), derived from 11-18 lung adenocarcinoma cells, which had been previously established in our laboratory (Table 3) ${ }^{25}$. While PC9 carries a deletion in exon 19 of EGFR, the 11-18 cells harbor the EGFR p.L858R.

Similarly to the PC9-derived models, four of the 11-18 resistant cell lines showed a significant increase of weak or moderate nuclear $\mathrm{pH} 3$ staining $(1+$ or $2+)$ in apparently resting cells. In contrast, the $\mathrm{H}$-scores for Ki67 and strong $(3+)$ $\mathrm{pH} 3$ staining in mitotic cells were not altered (Figs. 5a-c). Also, while parental 11-18 cells showed an IC50 for barasertib $>50 \mu \mathrm{M}$ in MTT assays, the AURKB inhibitor demonstrated a strong antiproliferative activity in 11-18-GR3 and GR5, with IC50s in the $\mathrm{nM}$ range (Fig. 5d, Table 3). 11-18-GR1, GR2, and GR6 cells showed intermediate sensitivity (IC50 10-20 $\mu \mathrm{M}$ ), and only 11-18-GR4 cells were completely resistant to the inhibitor. In order to gain insight into the mechanisms underlying these differences, cells were submitted to NGS, revealing the emergence of p.Q61 NRAS mutations in 11-18 GR1, GR2, GR4, and GR6 at allelic fractions of $4-22 \%$. In contrast, the two cell lines sensitive 
a

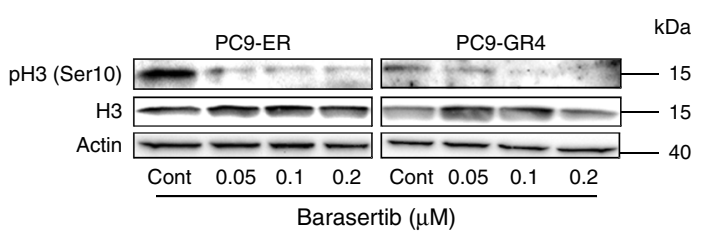

C

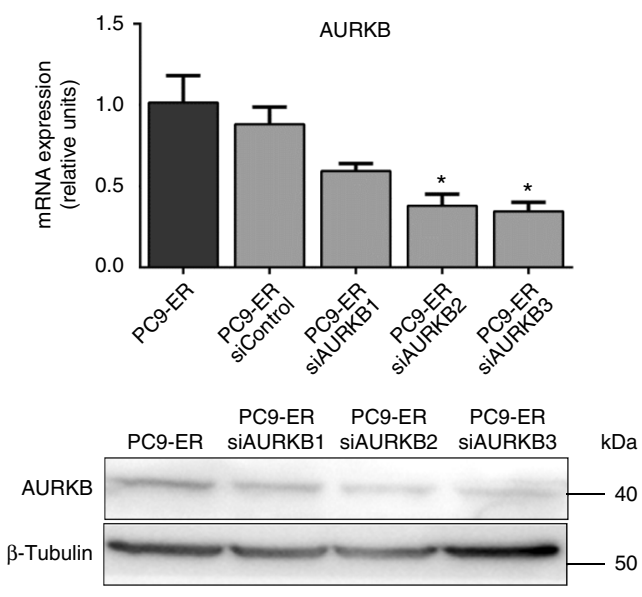

e

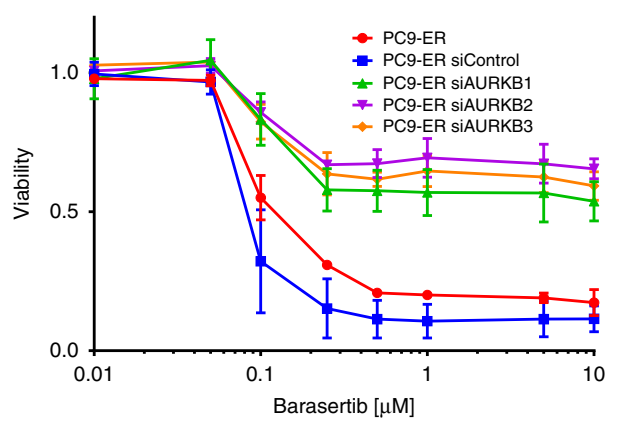

f
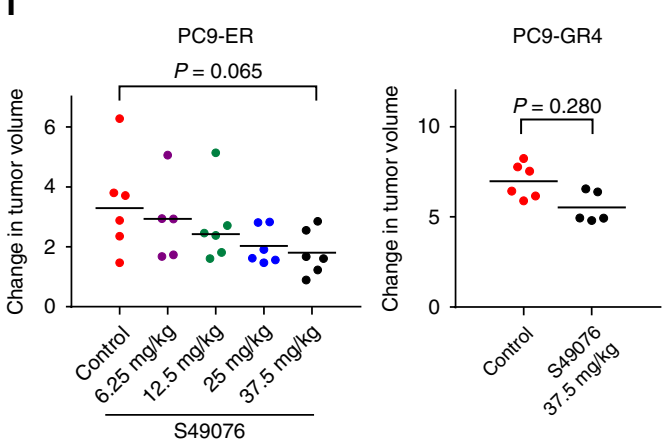

g

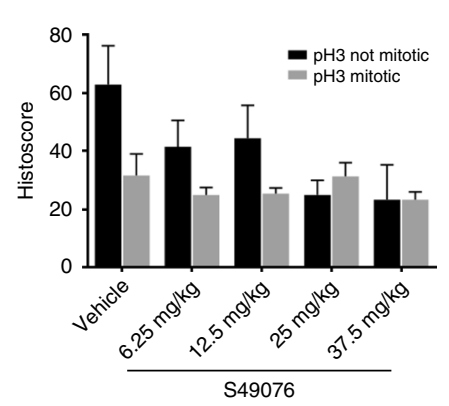

to barasertib (GR3 and GR5) did not show NRAS or other acquired mutations (Table 3). Remarkably, the NRAS gene showed a 4-5 fold amplification in the entire panel of 11-18 cells, independently of the presence of mutations.

The 11-18-GR2 (NRAS p.Q61L) and GR5 cells (wt for NRAS) were selected for further work. Similarly to PC9-derived lines, cells treated with barasertib appeared enlarged, vacuolized and multinucleated and comparison of direct counting vs. viability assays in 11-18-GR2 revealed a significant effect of barasertib on
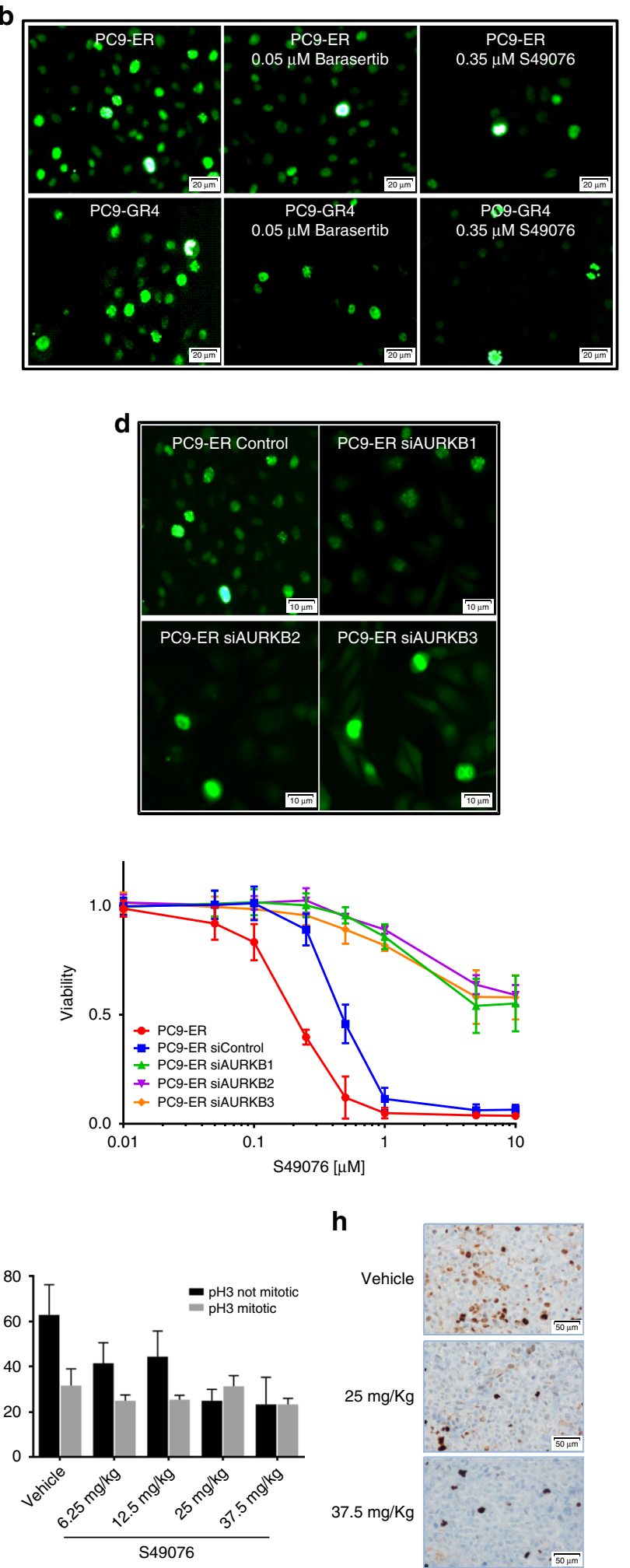

cell numbers, which was not reflected in MTT (Supplementary Fig. 10). Also, cell cycle analyses showed polyploidy followed by cell death exclusively $11-18$-GR5, while $\beta$-galactosidase staining demonstrated extensive senescence only in 11-18-GR2 (Figs. 5e-f).

pH3 increases in EGFR-mut NSCLC after progression to EGFR

TKIs. We used IHC to determine $\mathrm{pH} 3$ levels in 68 pretreatment 
Fig. 3 Inhibition and silencing of AURKB in cells and xenografts resistant to EGFR TKIs. a Western blot showing the dose-dependent decrease of pH3 levels induced by barasertib at $24 \mathrm{~h}$ in PC9-ER and PC9-GR4 cells. b ICC showing the inhibition of non-mitotic pH3 by barasertib and the MET/AXL/FGFR/ AURKB inhibitor S49076 after $24 \mathrm{~h}$ in PC9-ER and PC9-GR4 cells. Scale bars indicate $20 \mu \mathrm{m}$. c Levels of AURKB mRNA (upper panel) and protein (lower panel) in clones with partial silencing of the AURKB gene (PC9-ER siAURKB1-3), compared to parental PC9-ER and PC9-ER cells transfected with a control lentivirus (PC9-ER siControl). Results shown are means \pm SD of three independent determinations $(n=3)$ and asterisks indicate statistical significance $(p$ $<0.05$ ) in a two-sided Student's t-test. d ICC showing the inhibition of non-mitotic pH3 in clones with partial silencing of the AURKB gene. Scale bars indicate $10 \mu \mathrm{m}$. e Dose-response curves to barasertib (left) and S49076 (right) of the parental PC9-ER and the PC-ER clones with partial silencing of AURKB. Values shown are means \pm SD, experiments were conducted in tri $(n=3)$ or quadruplicates $(n=4)$. In each experiment, every concentration of drug was tested in sextuplicates $(n=6)$. $\mathbf{f}$ Effects of S49076 on the growth of subcutaneous PC9-ER (left panel) and PC9-GR4 (right panel) xenografts. Each point represents an individual tumor. Tumor dimensions were measured three times per week by digital calipers and volumes estimated according to the formula $V=\pi / 6 \times L \times W 2$, where $L$ is the long axis and $W$ is the short axis of tumor, respectively. Values are expressed as the fold-change in volume of each individual tumor after the 21 day treatment ( $n=6$ tumors per group). Lines indicated medians, and numbers correspond to levels of significance ( $p$ ) in a Mann-Whitman $\mathrm{U}$ test. $\mathbf{g} \mathrm{H}$-scores of mitotic and non-mitotic $\mathrm{pH} 3$ in the tumor xenografts. Values shown are means $\pm \mathrm{SD}(n=6$ tumors per group). $\mathrm{H}$ scores of every tumor were evaluated independently by two expert pathologists $(n=2)$. $\mathbf{h ~ p H 3 ~ i m m u n o s t a i n i n g ~ o f ~ r e p r e s e n t a t i v e ~ t u m o r ~ x e n o g r a f t s ~}$

tumor samples and 24 re-biopsies after relapse to EGFR TKIs from EGFR-mut patients (Figs. 6a-b). The H-score corresponding to mitotic $(3+) \mathrm{pH} 3$ did not show significant differences between both groups of samples $(p=0.135$ in a Mann-Whitney $U$ test), with medians of 15 in both cases. The score for Ki67 showed a similar behavior. In contrast, the median $\mathrm{H}$-score of non-mitotic $\mathrm{pH} 3$ was 48 for baseline samples vs. 75 for samples after progression $(p=0.021$ in a Mann-Whitney $U$ test $)$. Remarkably, only $7 \%$ of baseline biopsies had H-scores $>100$ for non-mitotic $\mathrm{pH} 3$ vs. $25 \%$ of re-biopsies $(p=0.023$ in a $\mathrm{Z}$ score test).

High pretreatment $\mathrm{pH} 3$ correlates with poor outcome. Finally, we investigated if $\mathrm{pH} 3$ was associated with patient outcome. Of the 68 patients with pretreatment samples mentioned above, 59 had a clinical data available and were used for the analysis (Supplementary Table 2). We found that a high total $\mathrm{pH} 3 \mathrm{H}$ score associated with poor response to EGFR TKIs and shorter overall survival. Of the 15 patients in the upper quartile, $9(60 \%)$ had progressive or stable disease; in contrast with 16/44 (36\%) for the rest of the cohort. Regarding overall survival, it was 15.7 months for the 15 patients with high $\mathrm{pH} 3$ vs. 37.6 months for the 44 remaining patients $(p=0.001$ in a long-rank test; $\mathrm{HR}=3.15$, CI $95 \%=1.98-13.11$ ) (Fig. 6c). When the same analysis was performed for Ki67, mitotic $\mathrm{pH} 3$ or non-mitotic $\mathrm{pH} 3$ separately, the differences in OS between the groups did not reach statistical significance (Supplementary Table 3).

\section{Discussion}

In this study, we used three panels of cell lines with acquired resistance EGFR TKIs; two were derived from PC9 cells, which carry a deletion in exon 19 of EGFR; and one from 11-18 cells, which harbor a p.L858R mutation. The eighteen cell lines of our panels presented a variety of molecular alterations, including acquisition or loss of the p.T790M, emergence of NRAS mutations, MET activation and AXL, EGFR, and FGFR1 upregulation. Previous reports have shown that EGFR-mut cell lines and tumors with acquired resistance to EGFR TKIs associated with AXL, MET, or FGFR1 are sensitive to inhibitors targeting those proteins ${ }^{11,30-32}$. In contrast, our PC9-derived cell lines did not show sensitivity in vitro to AXL, MET, and FGFR1 inhibitors and AXL silencing failed to abolish resistance to EGFR TKIs. However, GAS6 expression was widespread in our resistant cells, suggesting that AXL might play a role in the acquisition of resistance to EGFR TKIs that our relatively simple cell culture models failed to apprehend. In this respect, it has been demonstrated that dependency on AXL in certain cell line models can be easier to recapitulate in xenografts or $3 \mathrm{D}$ cultures $^{33,34}$. Regarding
MET, the cells in our panels with dysregulation did not show gene amplification or protein overexpression, only increased phosphorylation of the receptor under basal conditions; a fact that might explain their lack of response to MET inhibitors.

The resistant cell lines not carrying acquired mutations were strongly sensitive to barasertib, a mono-targeted AURKB inhibitor. Tozasertib, an AURKA+ AURKB inhibitor, and S49076, an inhibitor targeting AXL, MET, FGFR, and AURKB in Phase I/II clinical trials ${ }^{27,35}$, showed a similar behavior. Active AURKB phosphorylates histone $\mathrm{H} 3$, its main substrate, on Ser10. Despite the diversity of molecular alterations present in our resistant cell lines, we observed a widespread increase of $\mathrm{pH} 3$ levels not associated with a higher proliferation rate and circumscribed to the nuclei of apparently resting cells, where $\mathrm{pH} 3$ often appeared in foci. Such $\mathrm{pH} 3$ foci, often around nucleoli, have also been described in other cell types ${ }^{36}$. Partial silencing or drug inhibition of AURKB significantly decreased $\mathrm{pH} 3$ levels in apparently resting cells, indicating that activation of AURKB was the cause of the increase of $\mathrm{pH} 3$ in our panels of resistant cells. Amplification or overexpression of AURKB has been described in some solid tumors, where it has been correlated with poor prognosis ${ }^{18,20}$. However, gene expression and Western blotting experiments demonstrated that the activation of AURKB in our cell models of resistance was not associated with an upregulation at the mRNA or protein level. Also, the partial silencing of the AURKB gene did not re-sensitize cells to EGFR TKIs, suggesting that AURKB activation and increased $\mathrm{pH} 3$ levels were not responsible for the acquisition of resistance, but rather associated with it.

The mechanism responsible for increased AURKB activity in apparently non-mitotic cells remains to be elucidated. At early G2, AURKB is activated and extensively phosphorylates H3. After mitosis, AURKB undergoes targeted proteolysis by the anaphasepromoting complex/cyclosome (APC/C) ubiquitin ligase $\mathrm{l}^{37}$ and, during interphase, AURKB activity and $\mathrm{pH} 3$ levels remain low through mechanisms not fully understood ${ }^{38}$. At this respect, the phosphatases PP1 and PP2A can inactivate AURKB ${ }^{39}$, and AURKB activity and/or H3 Ser10 phosphorylation has been proposed to be actively suppressed by PP1 during interphase ${ }^{36}$. The observation that total AURKB levels in the parental and resistant cells are undistinguishable suggests that the increased AURKB activity and $\mathrm{pH} 3$ in the latter are a consequence of a deregulated dephosphorylation during interphase rather than impaired ubiquitination after mitosis.

Inhibition or silencing of AURKB has been demonstrated to induce $G 2 / M$ arrest and polyploidy in several cell line models ${ }^{28,40}$. Western blotting and flow-cytometry experiments showed that this was also the case in our EGFR-TKI-resistant cell lines, irrespective of the p.T790M or NRAS status. However, treatment with barasertib or S49076 had strong antitumor 
a

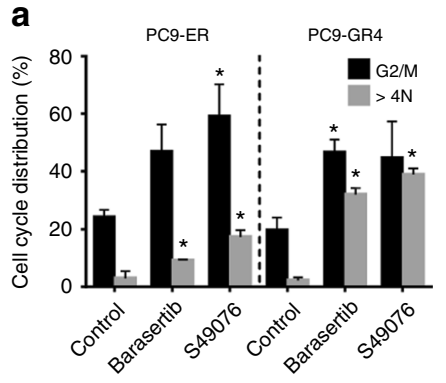

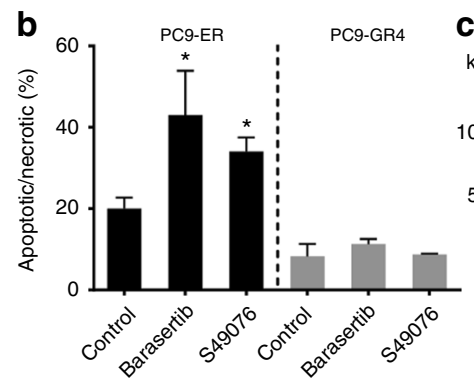

C

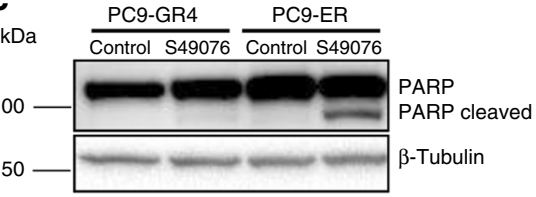

e

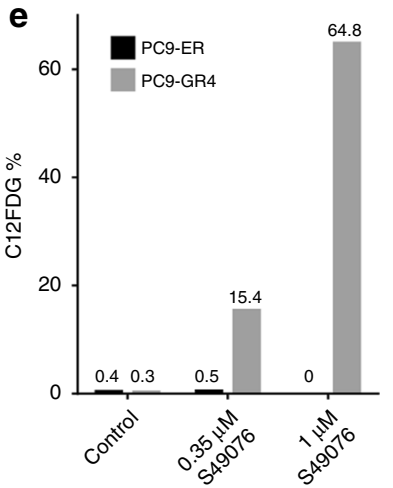

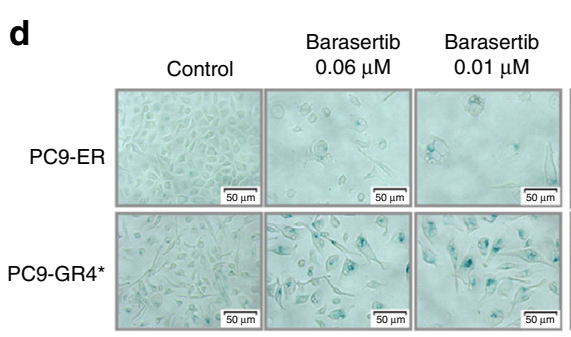

f
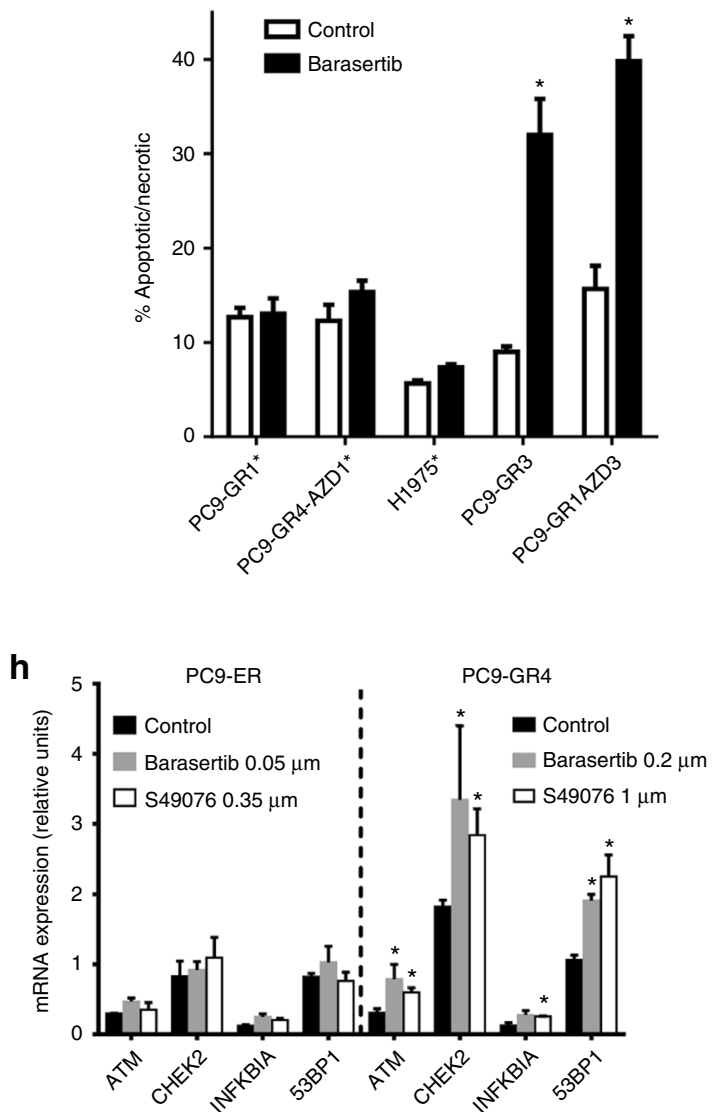

effects only in absence of p.T790M or NRAS mutations. Annexin $\mathrm{V}$ analyses revealed that both drugs induced apoptosis exclusively in p.T790M and NRAS-negative resistant cells. In positive cells, AURKB inhibition and polyploidy were followed by profound changes characteristic of the senescent phenotype, which were clearly observable after 2 days and included growth arrest, enlarged cellular size, cell flattening, irregular nuclei and overexpression of acidic $\beta$-galactosidase. However, if barasertib at $<0.2 \mu \mathrm{M}$ or $\mathrm{S} 49076$ at $<1 \mu \mathrm{M}$ were g

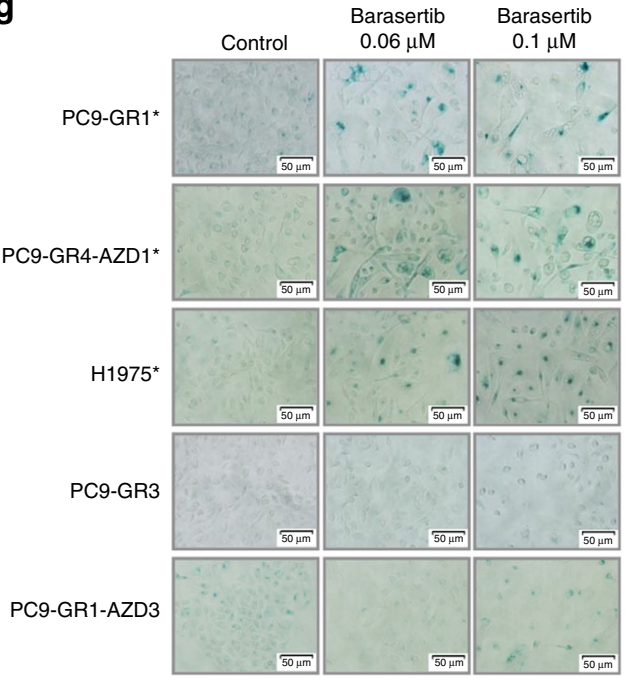

i

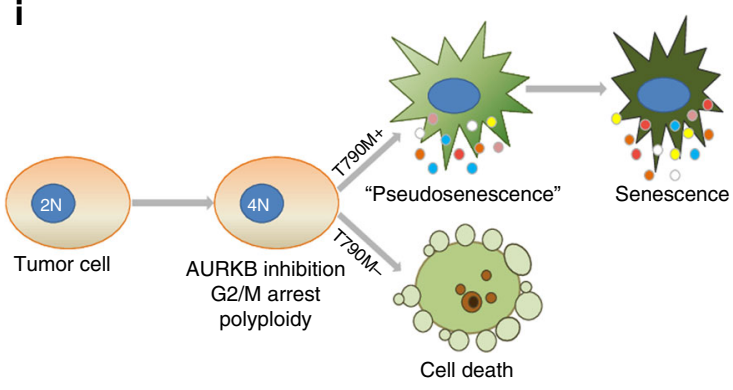

removed after less than 6 days, the p.T790M-positive PC9-GR4 resumed growth, indicating that $A U R K B$ inhibition initially induced a pseudo-senescent state where growth arrest was not yet irreversible ${ }^{29}$. In melanoma cells, AURKB has been shown to induce senescence through the DDR and the transcription factor $N F-\kappa B^{41}$. The up-regulation of $N F-\kappa B$ and the DDR genes $A T M, C H K-2$, and 53BP1 observed after treatment with barasertib and $\mathrm{S} 49076$ indicates that this is also the case in PC9-GR4. 
Fig. 4 Inhibition and silencing of AURKB in cell lines resistant to EGFR TKIs (cont). a Results of flow-cytometry experiments in PC9-ER (p.T790M negative) and PC9-GR4 (p.T790M positive) showing the induction of G2/M arrest and polyploidy after $24 \mathrm{~h}$ treatment with barasertib $(0.06 \mu \mathrm{M})$ or S49076 (0.35 $\mu \mathrm{M})$. Values shown are means \pm SD of three independent determinations $(n=3)$ and asterisks indicate statistical significance $(p<0.05)$ in a two-sided Student's t-test. b Results of flow-cytometry experiments showing the induction of cell death in PC9-ER but not in PC9-GR4 after $24 \mathrm{~h}$ treatment with barasertib $(0.06 \mu \mathrm{M})$ or $\mathrm{S} 49076(0.35 \mu \mathrm{M})$. Values shown are means \pm SD of three independent determinations $(n=3)$ and asterisks indicate statistical significance $(p<0.05)$ in a two-sided Student's t-test. c Western blot analysis of poly [ADP-ribose] polymerase 1 (PARP) cleavage in PC9-ER and PC9GR4 after $24 \mathrm{~h}$ with S49076 $(0.35 \mu \mathrm{M})$. d Acidic beta-galactosidase staining of PC9-ER and PC9-GR4 cells after $48 \mathrm{~h}$ with barasertib or S49076, showing the induction of senescence-associated activity in PC9-GR4. Scale bars indicate $50 \mu \mathrm{m}$. e Percentage of cells with active acidic beta-galactosidase, as measured by C12FDG fluorescent staining followed by flow cytometry, in PC9-ER and PC9-GR4 cells treated with S49076 for $48 \mathrm{~h}$. $\mathbf{f}$ Results of flowcytometry experiments of PC9-GR1, PC9-GR4-AZD1, H1975 (p.T790M positive), PC9-GR3, and PC9-GR1-AZD3 (p.T790M negative), showing the induction of apoptosis only in p.T790M-negative cells after $24 \mathrm{~h}$ treatment with barasertib $(0.06 \mu \mathrm{M})$. Values shown are means \pm SD of three independent determinations $(n=3)$ and asterisks indicate statistical significance $(p<0.05)$ in a two-sided Student's $t$-test. $\mathbf{g}$ Acidic beta-galactosidase staining of the same cells after $48 \mathrm{~h}$ with barasertib, showing the induction of senescence-associated activity exclusively in p.T790M-positive cells. Scale bars indicate 10 $\mu \mathrm{m}$. $\mathbf{h}$ Changes in the mRNA levels of selected genes after a 6-day treatment with S49076 or barasertib. Values shown are means \pm SD of three independent determinations $(n=3)$ and asterisks indicate statistical significance $(p<0.05)$ in a two-sided Student's $t$-test. i Proposed model to explain the effects of S49076 and barasertib in EGFR-TKI-resistant cells

\begin{tabular}{|c|c|c|c|c|c|c|c|c|c|c|c|}
\hline Cell line & $\begin{array}{l}\text { Doubling } \\
\text { time (h) }\end{array}$ & $\begin{array}{l}\text { EGFR } \\
\text { L858R }\end{array}$ & $\begin{array}{l}\text { EGFR } \\
\text { T790M }\end{array}$ & $\begin{array}{l}\text { EGFR } \\
\text { overexpression }\end{array}$ & $\begin{array}{l}\text { AXL } \\
\text { overexpression }\end{array}$ & $\begin{array}{l}\text { MET } \\
\text { activation }\end{array}$ & $\begin{array}{l}\text { FGFR1 } \\
\text { overexpression }\end{array}$ & $\begin{array}{l}\text { NRAS } \\
\text { amplification } \\
\text { (fold) }\end{array}$ & $\begin{array}{l}\text { NRAS } \\
\text { mutations } \\
\text { Q61L/R/K }\end{array}$ & $\begin{array}{l}\text { Gefitinib } \\
\text { (EGFR) } \\
\text { IC50 } \\
(\mu M)\end{array}$ & $\begin{array}{l}\text { Barasertib } \\
\text { (AURKB) } \\
\text { IC50 }(\mu \mathrm{M})\end{array}$ \\
\hline 11-18 & $23 \pm 4$ & + & - & - & - & - & - & 4 & - & 0.2 & $>50$ \\
\hline $11-18$ GR1 & $27 \pm 4$ & + & - & + & - & - & - & 5 & $\begin{array}{l}22 \% \\
\text { (Q61R) }\end{array}$ & 27.1 & 13 \\
\hline 11-18 GR2 & $25 \pm 2$ & + & - & + & - & + & - & 4 & $9 \%(\mathrm{Q} 61 \mathrm{~L})$ & 8.0 & $>50$ \\
\hline $11-18$ GR5 & $27 \pm 4$ & + & - & + & - & - & - & 3 & - & 20.8 & 0.06 \\
\hline $11-18$ GR6 & $22 \pm 1$ & + & - & + & ++ & - & - & 4 & 9\% (Q61K) & 8.1 & 18 \\
\hline
\end{tabular}

After growth arrest and polyploidy, AURKB inhibition triggers cell death in lung cancer, leukemia, prostate cancer, or neuroblastoma cell ${ }^{40,42,43}$ but senescence in fibroblasts, endothelial or melanoma cells ${ }^{41,44}$. In the case of fibroblasts, senescence is the result of cell cycle blockade by AURKB inhibition in presence of persistent strong mitogenic stimuli ${ }^{45}$. Similarly, it has been shown that aberrant replication or DNA damage, followed by growth arrest, leads to senescence in cells with oncogenes activated by mutations or other mechanisms ${ }^{46-49}$. In the context of resistance to TKIs, an AURKA/AURKB inhibitor has been reported to induce senescence in chronic myeloid leukemia cells carrying the imatinib-resistant T315I mutation in $A B L^{50}$. This could also be the case in our EGFR-TKI-resistant cells harboring acquired $\mathrm{p}$. T790M or NRAS mutations. These mutations induce persistent and strong mitogenic stimuli that, in presence of AURKB inhibition and cell cycle blockade, could trigger first a pseudosenescent and latter a truly senescent state. Senescent cells can survive for long periods of time, explaining the limited antitumor effects of S49076 or barasertib in p.T790M- or NRAS-positive cells. Conversely, in resistant cells without acquired mutations, cell cycle arrest in absence of oncogenic activation would induce cell death (Fig. 4i). Further support of this hypothesis comes from the observation that, in most p.T790M-negative lines of our PC9derived panels, a small fraction of cells (10-30\% in MTT assays) survives to micromolar concentrations of barasertib but not of S49076, showing a senescent phenotype (Table 2, Fig. 4d). We can speculate that, in resistant cells without acquired mutations, AXL, FGFR1, and EGFR overexpression or MET activation provide weak oncogenic stimuli. In presence of barasertib, which inhibits exclusively AURKB, these weak signals can direct a minor fraction of cells to senescence and survival. In contrast, being an MET/AXL/FGFR1/AURKB inhibitor, S49076 can suppress these stimuli and lead all cells to death.

The increase in $\mathrm{pH} 3$ associated with resistance to EGFR TKIs was not circumscribed to cell line models. In tumor samples from EGFR-mut NSCLC patients, the levels of non-mitotic $\mathrm{pH} 3$ were significantly elevated after progression to TKIs. In particular, $25 \%$ of tumors at progression had an $\mathrm{H}$-score $\geq 100$ vs. $7 \%$ at presentation. Similarly to resistant cell lines, increased non-mitotic $\mathrm{pH} 3$ coexisted with the p.T790M mutation, AXL overexpression or MET amplification. Finally, patients expressing high pretreatment levels of $\mathrm{pH} 3$ had a worse response rate to EGFR TKIs and a significantly shorter overall survival. These results were obtained in a relatively small number of samples. If confirmed in a larger cohort, they would indicate that AURKB activation and $\mathrm{pH} 3$ levels are involved in both intrinsic and acquired resistance to EGFR TKIs.

In summary, we have demonstrated that acquisition of resistance to EGFR TKIs in EGFR-mut cell line models and NSCLC patients is often associated with AURKB activation and increased levels of histone $\mathrm{H} 3$ phosphorylation. Inhibition of AURKB in these cell models leads to cell cycle arrest and polyploidy, followed by extensive cell death when the p.T790M or other acquired mutations are absent, or by senescence if they are present. Taken together, our results indicate that AURKB is a potential therapeutic target in NSCLC patients progressing on EGFR TKIs and not harboring resistance mutations.

\section{Methods}

Cell culture, proliferation assays, and cell cycle analyses. The H1975 cell line was purchased from the ATCC. Parental PC9 cells were provided by F. HoffmanLa Roche Ltd (Basel, Switzerland) with the authorization of Dr. Mayumi Ono 
a

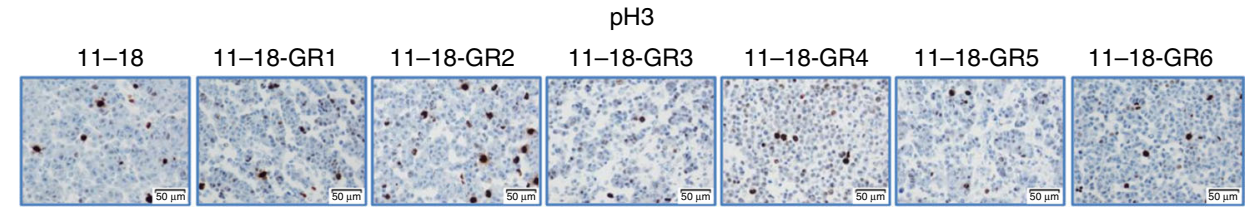

b

Ki67

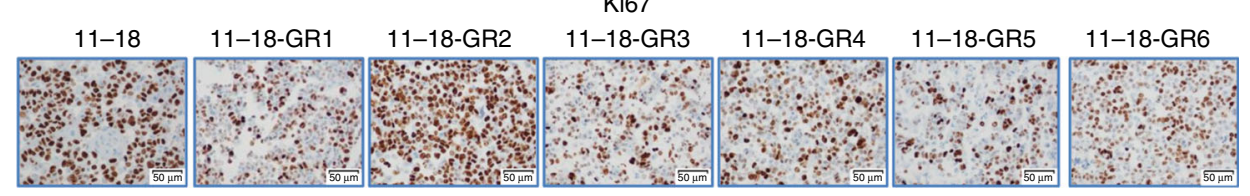

C
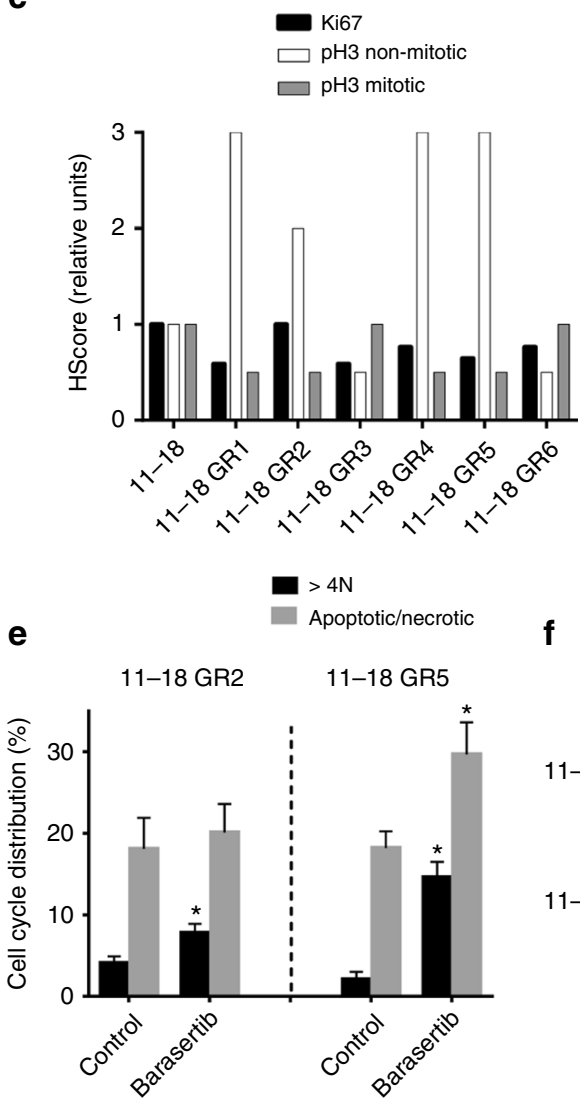

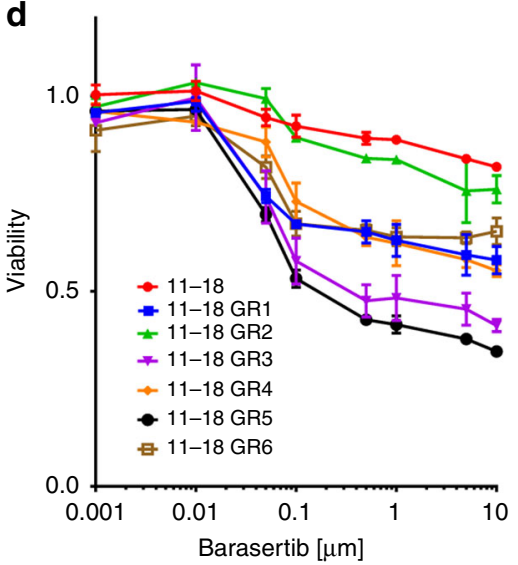

f

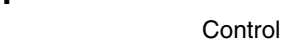

$11-18$ GR2

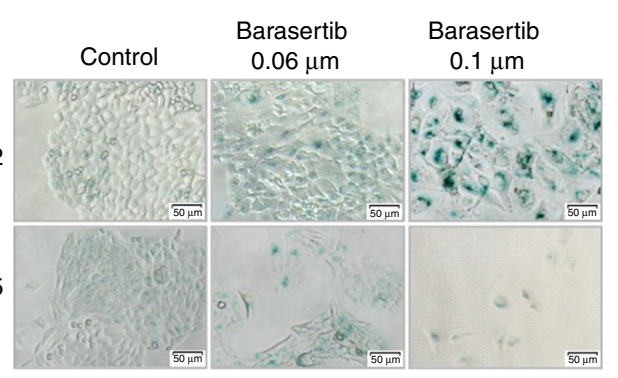

$11-18$ GR5

Fig. 5 Levels of $\mathrm{pH} 3$ and AURKB inhibition in 11-18 derived cell lines resistant to gefitinib. a-c $1 \mathrm{HC}$ of pH3 (a), Ki67 (b), and H-score (c) of Ki67, mitotic pH3 and non-mitotic $\mathrm{pH} 3$ in the six EGFR-mut cell lines resistant to EGFR TKIs. Scale bars indicate $50 \mu \mathrm{m}$. Values shown are means of two blind determinations performed independently by two expert pathologists $(n=2)$. d Dose-response curves of the parental and the EGFR-TKI-resistant cells to the AURKB inhibitor barasertib. Values shown are means $\pm S D$, experiments were conducted in tripicates $(n=3)$. In each experiment, every concentration of drug was tested in sextuplicates $(n=6)$. e Results of flow-cytometry experiments in 11-18-GR2 (NRAS Q61L) and GR5 (NRAS wt) showing polyploidy after $24 \mathrm{~h}$ treatment with barasertib $(0.1 \mu \mathrm{M})$ and apoptosis in 11-18-GR5 but not in GR2. Results shown are means \pm SD of three independent determinations $(n=3$ ) and asterisks indicate statistical significance $(p<0.05)$ in a two-sided Student's $t$-test. $\mathbf{f}$ Acidic beta-galactosidase staining of 11-18-GR2 and GR5 cells after $48 \mathrm{~h}$ with barasertib, showing the induction of senescence-associated activity in 11-18-GR2. Scale bars indicate $50 \mu \mathrm{m}$

(Kyushu University, Fukuoka, Japan). Parental 11-18 cells were kindly provided by Dr. Mayumi Ono. Resistant cells were derived by culturing parental cells with EGFR TKIs, starting at the IC50. Concentrations were increased in a stepwise way when proliferation resumed and cells could be trypsinized and re-plated. Fresh drug was added every $72-96 \mathrm{~h}$. Resistant cells were maintained as polyclonal populations under concentrations of drug $\geq 2.5 \mu \mathrm{M}$. Cell lines were authenticated by analyzing $>20$ polymorphisms by NGS (see below). In all cases, the genotypes and allelic fractions of parental and resistant cells were identical. S49076 was kindly provided by Servier (Suresnes, France). The rest of inhibitors were purchased from Selleck Chemicals (Houston, TX, USA) or MedChem Express (Monmouth Junction, NJ, USA).

Tumor cells were grown in a humidified atmosphere with $5 \% \mathrm{CO}_{2}$ at $37^{\circ} \mathrm{C}$ in RPMI1640 + 10\% fetal bovine serum (FBS), $50 \mu \mathrm{g} / \mathrm{mL}$ penicillin-streptomycin, and
$2 \mathrm{mM} \mathrm{L}$-Glutamine and routinely tested for mycoplasm contamination. For proliferation assays, cells were seeded at a density of 2000 (PC9) or 4000 (resistant) cells per well in 96-well plates, allowed to attach for $24 \mathrm{~h}$ and treated with drugs for $72 \mathrm{~h}$ unless otherwise indicated. For calculation of doubling times, cells were grown without drugs for $0,24,48$, and $72 \mathrm{~h}$ following attachment. After treatment, cells were incubated with medium containing $0.75 \mathrm{mg} / \mathrm{mL}$ of 3-(4,5-dimethylthiazol-2yl) $-2,5$-diphenyltetrazolium bromide (MTT) for $1-2 \mathrm{~h}$ at $37^{\circ} \mathrm{C}$, medium was removed and formazan crystals dissolved in $100 \mu \mathrm{L}$ DMSO. Cell numbers were estimated by measuring the absorbance at $495 \mathrm{~nm}$, using an Anthos 2020 microplate reader (Biochrom Ltd, Cambridge, UK). Data were derived from at least three independent experiments. In some experiments, direct counting in a hemocytometer after trypan blue staining was additionally used. For cell cycle analyses, cells were plated in T-25 flasks, allowed to attach, treated with drugs in 
a

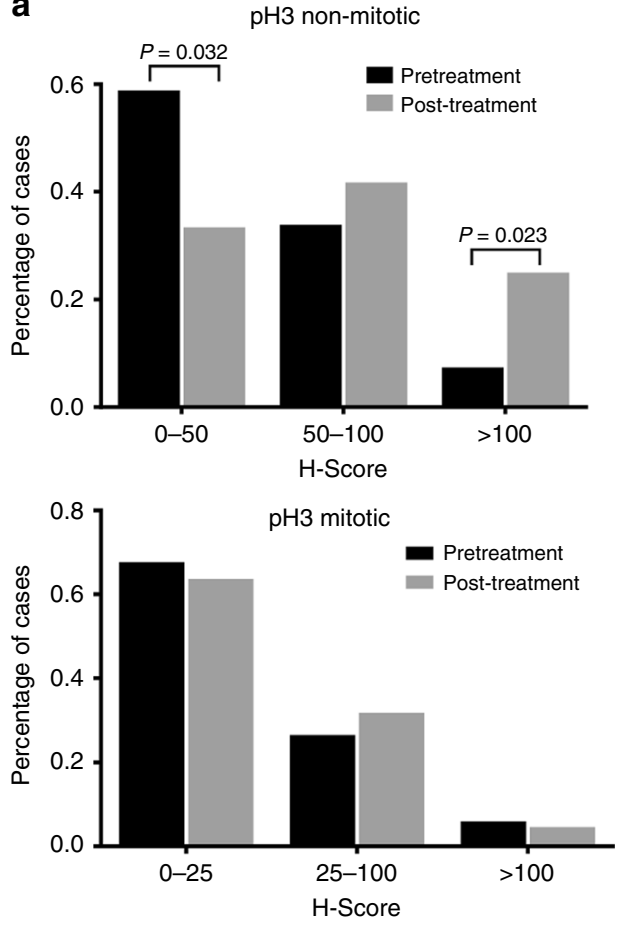

b

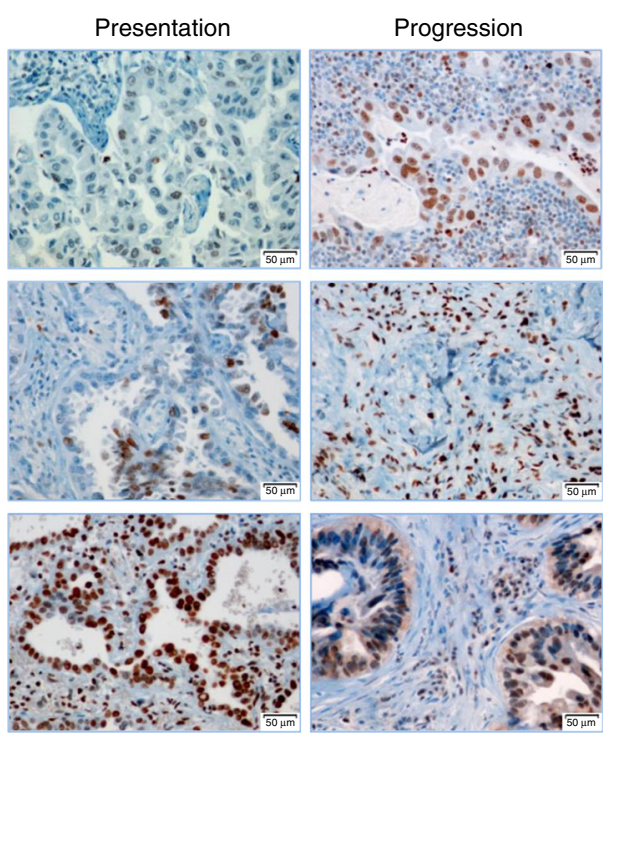

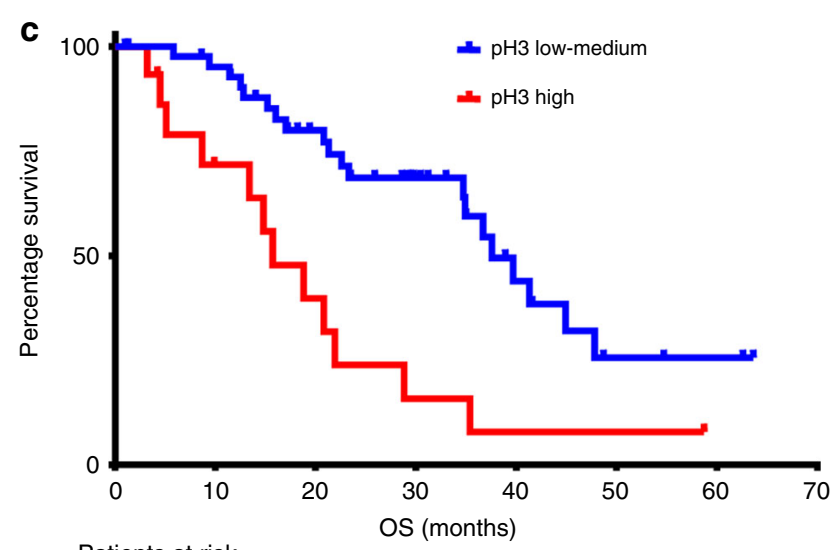

Patients at risk pH3 low-medium

$\begin{array}{cccccccc}\begin{array}{c}44 \\ \mathrm{pH} 3 \text { high }\end{array} & 40 & 29 & 19 & 9 & 4 & 3 & 1 \\ 15 & 10 & 6 & 3 & 2 & 2 & 1 & 1\end{array}$

Fig. $6 \mathrm{pH} 3$ in EGFR-mut NSCLC patients. a H-scores of non-mitotic (upper panel) and mitotic pH3 (lower panel) and in samples from EGFR-mut NSCLC patients baseline ( $n=68$, black bars) and after progression to EGFR TKIs ( $n=24$, gray bars). H-scores of every tumor were evaluated independently by two expert pathologists $(n=2)$. Numbers indicate levels of significance $(p)$ in a Z score test. $\mathbf{b} \mathrm{IHC}$ staining of pH3 of representative tumors at presentation and after progression to EGFR TKIs. Scale bars indicate $50 \mu \mathrm{m}$. c OS according to overall pH3 H-score at presentation in 59 EGFR-mut NSCLC patients treated with first-line EGFR TKIs. Median OS was 15.7 ( $\mathrm{Cl} 95 \%=8.9-22.5)$ months for the 15 patients with high pH3 (Q4, red line) vs. 37.6 months (Cl $95 \%=31.6-43.5)$ for the 44 patients with moderate or low pH3 (Q1-Q3, blue line) $(p=0.001$ in a two-sided log-rank test; $\mathrm{HR}=3.15, \mathrm{Cl} 95 \%=1.98-13.11)$

RPMI $+10 \%$ FBS, trypsinized, and centrifuged. Cell pellets were resuspended in PBS, fixed in $70 \%$ ethanol and incubated $\mathrm{o} / \mathrm{n}$ at $-20^{\circ} \mathrm{C}$. Fixed cells were subsequently centrifuged at $400 \times g$ for $5 \mathrm{~min}$ at $4{ }^{\circ} \mathrm{C}$, resuspended in $250 \mu \mathrm{L}$ of PBS with $50 \mu \mathrm{g} / \mathrm{mL}$ RNAse A (Sigma-Aldrich), incubated for $1 \mathrm{~h}$ at $37^{\circ} \mathrm{C}$, and stained with propidium iodide (PI) (Roche Diagnostics) for $30 \mathrm{~min}$ at room temperature.

Western blot, immunocytochemistry, and immunohistochemistry. For Western blotting, cells were seeded in T-75 flasks, allowed to attach o/n and treated for $2-24 \mathrm{~h}$ in RPMI $+10 \% \mathrm{FBS}$, unless otherwise indicated. Then, cultures were washed twice with cold PBS, scrapped into RIPA buffer (Cell Signaling Technology, Danvers, MO, USA) (20 mM Tris-HCl pH 7.5, $150 \mathrm{mM} \mathrm{NaCl}, 1 \mathrm{mM} \mathrm{Na}{ }_{2}$ EDTA, 1 mM EGTA, $1 \%$ NP- $40,1 \%$ sodium deoxycholate, $2.5 \mathrm{mM}$ sodium pyrophosphate, $1 \mathrm{mM} \beta$-glycerophosphate, $1 \mathrm{mM} \mathrm{Na} \mathrm{VO}_{4}, 1 \mu \mathrm{g} / \mathrm{ml}$ leupeptin, $2 \mathrm{mM}$ PMSF) with a protease inhibitors cocktail (Roche Diagnostics, Basel, Switzerland) and passed through an insulin syringe. Lysates were transferred to a microfuge tube, incubated on ice $15 \mathrm{~min}$, centrifuged for $10 \mathrm{~min}$ at $18,500 \times g$ and immediately analyzed or frozen at $-80^{\circ} \mathrm{C}$. Protein extracts $(25 \mu \mathrm{g})$ were boiled in Laemmly buffer (NuPAGE-LDS sample buffer $4 \times$; Invitrogen, Carlsbad, CA), resolved in SDSpolyacrylamide gels and transferred to a PVDF membrane (Bio-Rad, Hercules, $\mathrm{CA}$ ). Membranes were incubated for $1 \mathrm{~h}$ in Odyssey blocking buffer (Li-Cor Biosciences, Lincoln, NE) or Phosphoblocker reagent (Cell Biolabs Inc, San Diego, CA) for phosphorylated proteins. After blocking, membranes were cut, incubated with primary antibodies (Supplementary Table 4 ) o/n at $4{ }^{\circ} \mathrm{C}$, washed three times for 5 min each in PBS-Tween $0.1 \%$, and incubated for $2 \mathrm{~h}$ with secondary antibody (anti-rabbit or anti-mouse IgG horseradish peroxidase-conjugated secondary antibody; GE Healthcare, New York, NY). Finally, after three additional washes, membranes were incubated with Supersignal Chemiluminiscence substrate (Thermofisher Scientific, Waltham, MA, USA) and read with a a Bio-Rad ChemiDocMP Imaging System.

Fluorescent Immunocytochemistry (ICC) was performed on cells seeded in 24 well plates $\left(2.5 \times 10^{4}\right.$ per well $)$ with round cover slips and allowed to attach 
overnight. Cover slips were then removed, washed with PBS, fixed with $4 \%$ paraformaldehyde for $15 \mathrm{~min}$ and treated with cold methanol. After a wash in ICC buffer (PBS 1×, 0.1\% TX-100, 0.05\% Sodium azide, 0.5\% BSA), they were blocked in ICC $+10 \%$ FBS for 20 min at room temperature and incubated overnight at $4{ }^{\circ} \mathrm{C}$ with primary antibodies against the indicated proteins. Next, after three wash steps, Alexa Fluor 488-coupled secondary antibodies (Thermo Fisher Scientific) were added and incubated at room temperature for $2 \mathrm{~h}$. Finally, cover slips were washed three times with ICC, counterstained with DAPI, washed with water and mounted on glass slides with Vectashield (Vector Laboratories, Burlingame, CA, USA).

Immunohistochemistry (IHC) was performed on 5- $\mu \mathrm{m}$ sections of formalinfixed, paraffin embedded (FFPE) blocks using an automated immunostainer (BenchMark ULTRA, Ventana Medical Systems, Oro Valley, AZ, USA). Protein expression was quantified in a blind manner by two independent pathologists, using the histoscore ( $\mathrm{H}$-score) method, which evaluates the intensity of staining $(0$, non-staining; 1 , weak; 2 , median; or 3 , strong) and the percentage of positive cells. The antibodies used in this study are presented in the Supplementary Table 4 Histological images were obtained using a $\times 20$ objective, unless otherwise indicated.

Cell death and senescence assays. For cell death analyses, the Annexin-VFLUOS (An) staining kit (Roche Diagnostics) was employed, according to the manufacturer's instructions. Stained cells were analyzed with a FACSCanto II cytometer (BD Biosciences, Franklin Lakes, NJ) using the FACSDiva software version 6.1.2. The combination of Annexin V and PI was used to differentiate four cell populations; namely, viable cells (An-/PI-), early apoptotic (An+/PI-), necrotic (An-/PI+), and later apoptotic/necrotic $(\mathrm{An}+/ \mathrm{PI}+)$. For the initial senescence studies, cells were treated for $2 \mathrm{~h}$ to 8 days in 6 -well plates, and the Senescence Beta-galactosidase Staining kit (Cell Signaling) was be used to evaluate the presence of senescent cells by direct observation under the microscope. In subsequent studies, the percentage of senescent cells was quantified by flow cytometry. Cells $\left(3 \times 10^{4}\right)$ were grown in T-25 flasks and treated with selected drugs and incubated for $1 \mathrm{~h}$ with fresh RPMI $+10 \%$ FBS with $100 \mathrm{nM}$ bafilomycin A1 to neutralize the acidic $\mathrm{pH}$ of lysosomes. Finally, 5 -dodecanoylaminofluorescein di- $\beta$ D-galactopyranoside (C12FDG) was added at a final concentration of $33 \mu \mathrm{M}$ for 2 $\mathrm{h}$ and cells were submitted to flow cytometry using standard procedures. Gating strategies for flow-cytometry analyses of cell cycle, death and senescence are presented in Supplementary Fig. 11.

NGS, mRNA analysis, and gene silencing. Purified DNA $(16.5 \mu \mathrm{L})$ was used as a template for NGS analysis using the Gene Reader platform and GeneRead ${ }^{\circledast}$ QIAact Lung DNA Panel (QIAGEN, Hilden, Germany), according to manufacturer's instructions. The panel targets 549 variant positions in 17 selected genes frequently altered in lung tumors ( $A K T 1, A L K, B R A F, D D R 2, E G F R, E R B B 2 / H E R 2, E S R 1$, KIT, KRAS, MAP2K1, MET, NRAS, NTRK1, PDGFRA, PIK3CA, PTEN, ROS1) and detects copy number variations (CNV) in five genes (EGFR, FGFR1, ERBB2/HER2, MET, RICTOR).

RNA was isolated by standard procedures ${ }^{25}$. Primer and probe sets (Supplementary Table 5) were designed using Primer Express 3.0 Software (Applied Biosystems) according to their reference sequences (http://www.ncbi.nlm. nih.gov/LocusLink). Quantification of gene expression was performed using the ABI Prism 7900HT Sequence Detection System (Applied Biosystems). Expression levels were calculated using to the comparative $\Delta \Delta \mathrm{Ct}$ method $^{25}$. Commercial RNA controls were used as calibrators (Liver and Lung; Agilent Technologies, Santa Clara, CA, USA). For each cell line, a minimum of three independent experiments were performed.

Silencing of $A X L$ and $A U R K B$ was achieved by stable transfection using shRNA Lentiviral Transduction Particles (Sigma-Aldrich) or with pLV CRISPR-based lentivirus encoding Cas9, a puromycin-resistance gene and a sgRNA targeting $A U R K B$. Non-target shRNA or sgRNA particles were used as controls. Transfections were performed on 96-well plates by adding the particles (MOI $0.5-5)$ to exponentially growing, $50-70 \%$ confluent cells in presence of hexadimethrine bromide $(8 \mu \mathrm{g} / \mathrm{mL}$ ) (Sigma-Aldrich). After a $24 \mathrm{~h}$ incubation period, the viral particle-containing medium was replaced by fresh RPMI $+10 \%$ FBS. After an additional $24 \mathrm{~h}$, new medium was added, containing the appropriate concentration of puromycin (Sigma-Aldrich) for selection of transduced cells (1 $\mu \mathrm{g} / \mathrm{mL}$ ). Resistant colonies were picked up, expanded in medium with $1 \mu \mathrm{g} / \mathrm{mL}$ puromycin and assayed for the silencing of the target gene by western blotting and/ or mRNA expression analyses. Silenced clones were authenticated as described under cell culture.

Animal studies. All animal experiments were approved by the Ethical Committee of Animal Experimentation of the Parc Científic de Barcelona (PCB) following the guidance of the Association for Assessment and Accreditation of Laboratory Animal Care (AAALAC, Unit 1155). All relevant ethical regulations for animal testing and research were complied with. Mice were inoculated subcutaneously with $5 \times 10^{6}$ PC9-ER or GR4 cells in $0.3 \mathrm{~mL}$ of PBS with Matrigel. Treatments were started when the average tumor size reached $150-200 \mathrm{~mm}^{3}$ and mice were assigned into groups $(n=6)$ using randomized block design based upon their tumor volumes. S49076 was dissolved in $1 \%(w / v)$ Hydroxy Ethyl Cellulose (HEC) in
$100 \mathrm{mM}$ acetate buffer $\mathrm{pH} 4.5$ and orally administered twice a day with a maximum dose of $37.5 \mathrm{mg} / \mathrm{kg}$ bid. Tumor dimensions were measured blindly three times. At sacrifice, tumors were excised and included in paraffin for IHC analyses. The investigators were blinded for the evaluation of the results.

Patients. The patient cohort consisted of EGFR-mutated NSCLC patients diagnosed between 2006 and 2017 in the Dexeus Quirón University Hospital (Barcelona, Spain) and Fundación Santa Fe de Bogotá (Colombia). Studies were conducted in accordance with the Declaration of Helsinki and all relevant ethical regulations for work with human participants, under an approved protocol of the Institutional Review Board of Dexeus Quirón University Hospital. Samples were de-identified for patient confidentiality and informed written consent, also approved by the Institutional Review Board of Dexeus Quirón University, was obtained from all subjects.

Statistical analysis. The results were analyzed in GraphPad Prism v6.0 using appropriate statistical tests. $p$-values of $<0.05$ were considered to be statistically significant.

Reporting summary. Further information on experimental design is available in the Nature Research Reporting Summary linked to this article.

\section{Data availability}

The NGS data generated in this study have been deposited in the in the Sequence Read Archive (SAR) of the National Center for Biotechnology Information (NCBI), under the accession codes PRJNA524804 (project) and SAMN11035311-SAMN11035324 (individual samples). The source data underlying Figs. 1e, 2a, 2g, 3c, 3e-g, 4a, 4b, 4e, 4f, $4 \mathrm{~h}, 5 \mathrm{c}-\mathrm{e}, 6 \mathrm{a}, 6 \mathrm{c}$ and Supplementary Figs. 1b, 2, 3c, 3d, 4a, 4b, 5b, 5c, 6a, 6c, 7b, 7c, 9a, 9b, $10 \mathrm{~b}, 10 \mathrm{c}$ are provided as a source data file. The file contains uncropped and unprocessed scans of the western blots presented in Figs. $1 b, 2 c, 2 d$ and $3 a$. The source data file has also been deposited in the Open Science Framework (OSF) repository under the unique identifier DOI 10.17605/OSF.IO/JW4C7. The authors declare that all other data supporting the findings of this study are available within the main article and its Supplementary Information file or from corresponding authors upon reasonable request. A reporting summary for this article is available as Supplementary Information file.

Received: 20 August 2018 Accepted: 28 March 2019 Published online: 18 April 2019

\section{References}

1. Ferlay, J. et al. Cancer incidence and mortality worldwide: sources, methods and major patterns in GLOBOCAN 2012. Int. J. Cancer 136, E359-E386 (2015).

2. Rosell, R. et al. Screening for epidermal growth factor receptor mutations in lung cancer. N. Engl. J. Med. 361, 958-967 (2009).

3. Huang, S. F. et al. High frequency of epidermal growth factor receptor mutations with complex patterns in non-small cell lung cancers related to gefitinib responsiveness in Taiwan. Clin. Cancer Res. 10, 8195-8203 (2004).

4. Maemondo, M. et al. Gefitinib or chemotherapy for non-small-cell lung cancer with mutated EGFR. N. Engl. J. Med. 362, 2380-2388 (2010).

5. Rosell, R. et al. Erlotinib versus standard chemotherapy as first-line treatment for European patients with advanced EGFR mutation-positive non-small-cell lung cancer (EURTAC): a multicentre, open-label, randomised phase 3 trial. Lancet Oncol. 13, 239-246 (2012).

6. Wu, Y. L. et al. Afatinib versus cisplatin plus gemcitabine for first-line treatment of Asian patients with advanced non-small-cell lung cancer harbouring EGFR mutations (LUX-Lung 6): an open-label, randomised phase 3 trial. Lancet Oncol. 15, 213-222 (2014).

7. Kobayashi, S. et al. EGFR mutation and resistance of non-small-cell lung cancer to gefitinib. N. Engl. J. Med. 352, 786-792 (2005).

8. Tan, C. S., Gilligan, D. \& Pacey, S. Treatment approaches for EGFR-inhibitorresistant patients with non-small-cell lung cancer. Lancet Oncol. 16, e447-e459 (2015)

9. Xie, S. et al. Mer receptor tyrosine kinase is frequently overexpressed in human non-small cell lung cancer, confirming resistance to erlotinib. Oncotarget 6, 9206-9219 (2015).

10. Koch, H., Busto, M. E., Kramer, K., Medard, G. \& Kuster, B. Chemical proteomics uncovers EPHA2 as a mechanism of acquired resistance to small molecule EGFR kinase inhibition. J. Proteome. Res. 14, 2617-2625 (2015).

11. Zhang, Z. et al. Activation of the AXL kinase causes resistance to EGFRtargeted therapy in lung cancer. Nat. Genet. 44, 852-860 (2012).

12. Mok, T. S. et al. Osimertinib or platinum-pemetrexed in EGFR T790Mpositive lung cancer. N. Engl. J. Med. 376, 629-640 (2017). 
13. Chic, N., Mayo-de-Las-Casas, C. \& Reguart, N. Successful treatment with gefitinib in advanced non-small cell lung cancer after acquired resistance to osimertinib. J. Thorac. Oncol. 12, e78-e80 (2017).

14. Thress, K. S. et al. Acquired EGFR C797S mutation mediates resistance to AZD9291 in non-small cell lung cancer harboring EGFR T790M. Nat. Med. 21, 560-562 (2015).

15. Ortiz-Cuaran, S. et al. Heterogeneous mechanisms of primary and acquired resistance to third-generation EGFR inhibitors. Clin. Cancer Res. 22, 4837-4847 (2016).

16. Yang, J. et al. AZD1152, a novel and selective Aurora B kinase inhibitor, induces growth arrest, apoptosis, and sensitization for tubulin depolymerizing agent or topoisomerase II inhibitor in human acute leukemia cells in vitro and in vivo. Blood 110, 2034-2040 (2007).

17. Wilkinson, R. W. et al. AZD1152, a selective inhibitor of Aurora B kinase, inhibits human tumor xenograft growth by inducing apoptosis. Clin. Cancer Res. 13, 3682-3688 (2007).

18. Tang, A. et al. Aurora kinases: novel therapy targets in cancers. Oncotarget 8, 23937-23954 (2017).

19. Ditchfield, C. et al. Aurora B couples chromosome alignment with anaphase by targeting BubR1, Mad2, and Cenp-E to kinetochores. J. Cell Biol. 161, 267-280 (2003).

20. Takeshita, M. et al. Aurora-B overexpression is correlated with aneuploidy and poor prognosis in non-small cell lung cancer. Lung Cancer 80, 85-90 (2013).

21. Hole, S., Pedersen, A. M., Lykkesfeldt, A. E. \& Yde, C. W. Aurora kinase A and $\mathrm{B}$ as new treatment targets in aromatase inhibitor-resistant breast cancer cells. Breast Cancer Res. Treat. 149, 715-726 (2015).

22. Al-Khafaji, A. S. et al. Aurora B expression modulates paclitaxel response in non-small cell lung cancer. Br. J. Cancer 116, 592-599 (2017).

23. Hoellein, A. et al. Aurora kinase inhibition overcomes cetuximab resistance in squamous cell cancer of the head and neck. Oncotarget 2, 599-609 (2011).

24. Phadke, M. S., Sini, P. \& Smalley, K. S. The novel ATP-competitive MEK/ Aurora kinase inhibitor BI-847325 overcomes acquired BRAF Inhibitor resistance through suppression of Mcl-1 and MEK expression. Mol. Cancer Ther. 14, 1354-1364 (2015).

25. Jacobsen, K. et al. Convergent Akt activation drives acquired EGFR inhibitor resistance in lung cancer. Nat. Commun. 8, 410 (2017).

26. Myers, S. H., Brunton, V. G. \& Unciti-Broceta, A. AXL inhibitors in cancer: a medicinal chemistry perspective. J. Med. Chem. 59, 3593-3608 (2016).

27. Burbridge, M. F. et al. S49076 is a novel kinase inhibitor of MET, AXL, and FGFR with strong preclinical activity alone and in association with bevacizumab. Mol. Cancer Ther. 12, 1749-1762 (2013).

28. Clemenson, C. et al. The MET/AXL/FGFR inhibitor S49076 impairs aurora B activity and improves the antitumor efficacy of radiotherapy. Mol. Cancer Ther. 16, 2107-2119 (2017).

29. Loaiza, N. \& Demaria, M. Cellular senescence and tumor promotion: is aging the key? Biochim. Biophys. Acta 1865, 155-167 (2016).

30. Engelman, J. A. et al. MET amplification leads to gefitinib resistance in lung cancer by activating ERBB3 signaling. Science 316, 1039-1043 (2007).

31. Terai, H. et al. Activation of the FGF2-FGFR1 autocrine pathway: a novel mechanism of acquired resistance to gefitinib in NSCLC. Mol. Cancer Res. 11, 759-767 (2013).

32. Scagliotti, G. et al. Phase III multinational, randomized, double-blind, placebo-controlled study of tivantinib (ARQ 197) plus erlotinib versus erlotinib alone in previously treated patients with locally advanced or metastatic nonsquamous non-small-cell lung cancer. J. Clin. Oncol. 33, 2667-2674 (2015).

33. Linger, R. M. et al. Mer or Axl receptor tyrosine kinase inhibition promotes apoptosis, blocks growth and enhances chemosensitivity of human non-small cell lung cancer. Oncogene 32, 3420-3431 (2013).

34. Leconet, W. et al. Therapeutic activity of anti-AXL antibody against triplenegative breast cancer patient-derived xenografts and metastasis. Clin. Cancer Res. 23, 2806-2816 (2017).

35. Rodon, J. et al. First-in-human phase I study of oral S49076, a unique MET/ AXL/FGFR inhibitor, in advanced solid tumours. Eur. J. Cancer 81, 142-150 (2017).

36. Hayashi-Takanaka, Y., Yamagata, K., Nozaki, N. \& Kimura, H. Visualizing histone modifications in living cells: spatiotemporal dynamics of $\mathrm{H} 3$ phosphorylation during interphase. J. Cell Biol. 187, 781-790 (2009).

37. Lindon, C., Grant, R. \& Min, M. Ubiquitin-mediated degradation of aurora kinases. Front. Oncol. 5, 307 (2015).

38. Lee, S. et al. IK-guided PP2A suppresses Aurora B activity in the interphase of tumor cells. Cell. Mol. Life Sci. 73, 3375-3386 (2016).

39. Sugiyama, K. et al. Aurora-B associated protein phosphatases as negative regulators of kinase activation. Oncogene 21, 3103-3111 (2002).
40. Xie, H. et al. Identification of an Aurora kinase inhibitor specific for the Aurora B isoform. Cancer Res. 73, 716-724 (2013).

41. Liu, Y. et al. Targeting aurora kinases limits tumour growth through DNA damage-mediated senescence and blockade of NF-kappaB impairs this druginduced senescence. EMBO Mol. Med. 5, 149-166 (2013).

42. Bogen, D. et al. Aurora B kinase is a potent and selective target in MYCNdriven neuroblastoma. Oncotarget 6, 35247-35262 (2015).

43. Zekri, A. et al. AZD1152-HQPA induces growth arrest and apoptosis in androgen-dependent prostate cancer cell line ( $\mathrm{LNCaP})$ via producing aneugenic micronuclei and polyploidy. Tumour Biol. 36, 623-632 (2015).

44. Kim, H. J., Cho, J. H., Quan, H. \& Kim, J. R. Down-regulation of Aurora B kinase induces cellular senescence in human fibroblasts and endothelial cells through a p53-dependent pathway. FEBS Lett. 585, 3569-3576 (2011).

45. Sadaie, M. et al. Cell-based screen for altered nuclear phenotypes reveals senescence progression in polyploid cells after Aurora kinase B inhibition. Mol. Biol. Cell 26, 2971-2985 (2015).

46. Serrano, M., Lin, A. W., McCurrach, M. E., Beach, D. \& Lowe, S. W. Oncogenic ras provokes premature cell senescence associated with accumulation of p53 and p16INK4a. Cell 88, 593-602 (1997).

47. Bartkova, J. et al. Oncogene-induced senescence is part of the tumorigenesis barrier imposed by DNA damage checkpoints. Nature 444, 633-637 (2006).

48. Di Micco, R. et al. Oncogene-induced senescence is a DNA damage response triggered by DNA hyper-replication. Nature 444, 638-642 (2006).

49. Michaloglou, C. et al. BRAFE600-associated senescence-like cell cycle arrest of human naevi. Nature 436, 720-724 (2005).

50. Wang, L. X. et al. Aurora A kinase inhibitor AKI603 induces cellular senescence in chronic myeloid leukemia cells harboring T315I mutation. Sci. Rep. 6, 35533 (2016).

\section{Acknowledgements}

Financial support for this study was provided by the Institut de Recherches Internationales Servier (Suresnes, France)

\section{Author contributions}

J.B.A., V.C., M.S., F.C., M.B., Ra.R. and M.A.M.V. conceived and designed the study. J.B. A., J.C.S., A.G.C., S.R., C.T., Ru.R., J.C., S.G.R., C.C.S. and M.A.M.V. conducted the experiments, acquired, and analyzed data. S.V., A.F.C. and N.K. provided clinical samples and collected clinical information. J.B.A., V.C., M.S., J.C.S., S.G.R., Ra.R. and M.A.M.V. wrote, reviewed, and/or revised the manuscript.

\section{Additional information}

Supplementary Information accompanies this paper at https://doi.org/10.1038/s41467 019-09734-5.

Competing interests: V.C., M.S., F.C. and M.B. were employees of Servier at the time of this study. The remaining authors declare no competing interests.

Reprints and permission information is available online at http://npg.nature.com/ reprintsandpermissions/

Journal peer review information: Nature Communications thanks David Barbie and the other anonymous reviewer for their contribution to the peer review of this work. Peer reviewer reports are available.

Publisher's note: Springer Nature remains neutral with regard to jurisdictional claims in published maps and institutional affiliations.

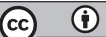

Open Access This article is licensed under a Creative Commons Attribution 4.0 International License, which permits use, sharing, adaptation, distribution and reproduction in any medium or format, as long as you give appropriate credit to the original author(s) and the source, provide a link to the Creative Commons license, and indicate if changes were made. The images or other third party material in this article are included in the article's Creative Commons license, unless indicated otherwise in a credit line to the material. If material is not included in the article's Creative Commons license and your intended use is not permitted by statutory regulation or exceeds the permitted use, you will need to obtain permission directly from the copyright holder. To view a copy of this license, visit http://creativecommons.org/ licenses/by/4.0/

(C) The Author(s) 2019 